\title{
D3-D7 quark-gluon plasmas at finite baryon density
}

\author{
Francesco Bigazzi, ${ }^{a}$ Aldo L. Cotrone, ${ }^{b}$ Javier Mas, ${ }^{c}$ Daniel Mayerson ${ }^{d}$ and \\ Javier Tarrío ${ }^{e}$ \\ ${ }^{a}$ Dipartimento di Fisica e Astronomia, Università di Firenze and INFN Sezione di Firenze, \\ Via G. Sansone 1, I-50019 Sesto Fiorentino (Firenze), Italy \\ ${ }^{b}$ Dipartimento di Fisica, Università di Torino and INFN Sezione di Torino, \\ Via P. Giuria 1, I-10125 Torino, Italy \\ ${ }^{c}$ Departamento de Física de Partículas, Universidade de Santiago de Compostela and Instituto \\ Galego de Física de Altas Enerxías (IGFAE), E-15782, Santiago de Compostela, Spain \\ ${ }^{d}$ Institute for theoretical physics, K.U. Leuven, \\ Celestijnenlaan 200D, B-3001 Leuven, Belgium \\ e Institute for Theoretical Physics, Universiteit Utrecht, \\ 3584 CE, Utrecht, The Netherlands \\ E-mail: bigazzi@fi.infn.it, cotrone@to.infn.it, javier.mas@usc.es, \\ drm56@cam.ac.uk, 1.j.tarriobarreiro@uu.nl
}

Abstract: We present the string dual to $\mathrm{SU}\left(N_{c}\right) \mathcal{N}=4 \mathrm{SYM}$, coupled to $N_{f}$ massless fundamental flavors, at finite temperature and baryon density. The solution is determined by two dimensionless parameters, both depending on the 't Hooft coupling $\lambda_{h}$ at the scale set by the temperature $T: \epsilon_{h} \sim \lambda_{h} N_{f} / N_{c}$, weighting the backreaction of the flavor fields and $\tilde{\delta} \sim \lambda_{h}^{-1 / 2} n_{b} /\left(N_{f} T^{3}\right)$, where $n_{b}$ is the baryon density. For small values of these two parameters the solution is given analytically up to second order. We study the thermodynamics of the system in the canonical and grand-canonical ensembles. We then analyze the energy loss of partons moving through the plasma, computing the jet quenching parameter and studying its dependence on the baryon density. Finally, we analyze certain "optical" properties of the plasma. The whole setup is generalized to non abelian strongly coupled plasmas engineered on D3-D7 systems with D3-branes placed at the tip of a generic singular Calabi-Yau cone. In all the cases, fundamental matter fields are introduced by means of homogeneously smeared D7-branes and the flavor symmetry group is thus a product of abelian factors.

KEYWORDS: Gauge-gravity correspondence, Holography and quark-gluon plasmas

ArXiv EPRINT: 1101.3560 


\section{Contents}

1 Introduction and summary 1

2 Ansatz and effective Lagrangian 3

$\begin{array}{llr}3 & \text { The perturbative solution } & 6\end{array}$

4 Physical properties of the dual plasmas $\quad 10$

$\begin{array}{ll}4.1 \text { Thermodynamics } & 10\end{array}$

$\begin{array}{lll}4.2 & \text { Thermodynamical potentials } & 11\end{array}$

$\begin{array}{lll}\text { 4.2.1 Canonical ensemble } & 11\end{array}$

$\begin{array}{lll}\text { 4.2.2 Grand-canonical ensemble } & 13\end{array}$

$\begin{array}{lll}4.3 & \text { Probe parton energy loss } & 15\end{array}$

$\begin{array}{ll}\text { 4.3.1 Comparison schemes } & 16\end{array}$

$\begin{array}{ll}4.4 \text { Remarks on "optical" properties } & 17\end{array}$

$\begin{array}{lll}5 & \text { Conclusions } & 19\end{array}$

A Technical details $\quad 20$

A.1 Equations of motion from the ten dimensional action 20

A.2 Solution with explicit UV cutoff 22

\section{Introduction and summary}

Heavy ion collision experiments at RHIC and LHC allow us to explore a relevant corner of the QCD phase diagram (high temperature and relatively small baryon chemical potential), where the theory is expected to be deconfined. Both the results collected during the tenyear run of RHIC [1-4] and the preliminary ones at LHC [5-7] actually indicate that a quark-gluon "fireball" is formed and behaves like a strongly coupled system: a liquid with very small viscosity over entropy density ratio. Holographic methods provide interesting tools to analyze these kind of systems. The simplest and best studied example is the conformal $\mathcal{N}=4 \mathrm{SYM}$ plasma which, unexpectedly, has proven to share some properties with the QCD one. This fact has stimulated further research works with the aim of refining this master holographic model, for example by adding fundamental matter fields. The latter has been performed mainly in the quenched approximation.

In [8] some of the authors have presented a ten dimensional black-hole solution dual to the non conformal plasma of $\mathcal{N}=4 \mathrm{SYM}$ coupled to $N_{f} \gg 1$ massless flavors. ${ }^{1}$ The latter were introduced by means of homogeneously smeared D7-branes [11-13], extended

\footnotetext{
${ }^{1}$ All the hydrodynamic transport coefficients of the model were derived in $[9,10]$.
} 
along the radial direction up to the black hole horizon. The smearing reduces the flavor symmetry group to a product of abelian factors and allows a simple way to account for the backreaction of the D7-branes and thus to explore the "unquenched" regime in the dual field theory. ${ }^{2}$ The analysis was also generalized to $\mathcal{N}=1$ non abelian plasmas engineered on D3-D7 systems with D3-branes placed at the tip of a generic singular Calabi-Yau cone. In the zero temperature limit, the resulting backgrounds coincide with those found in $[28] .^{3}$

In the present paper we extend the above construction to include a finite baryon density (or chemical potential, in the alternative thermodynamical ensemble) for the flavor fields. Working on this problem with holographic techniques is especially interesting, taking also into account that there is no systematic way of dealing with finite baryon density in strongly coupled QCD (lattice QCD suffering from the so-called sign problem).

We provide a novel gravity solution, dual to the above class of flavored plasmas in the planar limit at strong 't Hooft coupling. While the equations of motion we derive are completely general, the solution can be given in closed analytic form up to second order in $\epsilon_{h} \sim \lambda_{h} N_{f} / N_{c}$ (where $\lambda_{h}$ is the 't Hooft coupling at the temperature $T$ of the plasma) and $\tilde{\delta} \sim \lambda_{h}^{-1 / 2} n_{b} /\left(N_{f} T^{3}\right)$, where $n_{b}$ is the baryon density. The gauge theories we focus on become pathological at some UV scale, developing a Landau pole. This is signaled, for example, by a running dilaton (accounting for the breaking of conformal invariance induced by the flavor fields) blowing up at a finite radial value. Correspondingly, the dual gravity solutions are not reliable close to that scale. Keeping $\epsilon_{h}$ small allows both to focus on a regime where the solutions are reliable and to decouple the IR physics - which is the regime we focus on - from the pathological UV behavior.

We also consider the regime of non-large baryon density, $\tilde{\delta} \ll 1$, both because it is the relevant regime for the RHIC and LHC experiments and because it allows to derive an analytic solution. Exploring the $\tilde{\delta} \sim 1$ regime requires a numerical analysis, that we plan to provide in the near future.

The main results and the outline of the paper are as follows. In section 2 we present the action and the ansatz for the D3-D7 setup at finite baryon density. A set of second order differential equations is given in terms of the functions of the radial variable appearing in the ansatz. In section 3 we solve the equations analytically, in a perturbative expansion in $\epsilon_{h}$ and $\tilde{\delta}$ up to second order in both parameters. In section 4 we perform the study of the thermodynamics of the system in the canonical and grand-canonical ensembles, checking the (non-trivial) closure of the various thermodynamic relations. We then explore the effects of the baryon number density on the energy loss of probes through the plasma, in particular on the jet quenching parameter. While the overall effect of flavors is to enhance the jet quenching [8], the effect of finite baryon density depends on the specific choice of comparison scheme of different theories. We finally provide some considerations on certain "optical" properties of the plasma, thinking about the possible gauging of the global U(1). Section 5 contains some concluding remarks. We also provide an appendix with some details on the ten-dimensional action, the equations of motion, the Bianchi identities and their solutions.

\footnotetext{
${ }^{2}$ For other holographic studies of thermal unquenched flavors, see [14-27].

${ }^{3}$ Other solutions employing the smearing technique appear in [29-53].
} 
The backgrounds we provide correspond to charged black holes in (slightly deformed) AdS, the charge being dual to a finite baryon density. The regime of validity is completely specified and the solution is totally reliable in that regime - there are no uncontrolled approximations. It is the first solution of this kind in the literature and thus it is suitable for the study of a number of physical effects of the baryon density. We hope to explore further the physics of this system in the future.

\section{Ansatz and effective Lagrangian}

The field theories we focus on are realized on the $4 \mathrm{~d}$ intersection of $N_{c}$ "color" D3 and $N_{f}$ homogeneously smeared "flavor" D7-branes. The D3-branes are placed at the tip of a Calabi-Yau (CY) cone over a Sasaki-Einstein manifold $X_{5}$, the latter being a U(1) fiber bundle over a four dimensional Kähler-Einstein (KE) base. The ambient spacetime, a product of $4 \mathrm{~d}$ Minkowski and the CY cone, will be deformed by the backreaction of both kind of branes which respectively source a (self dual) $F_{5}$ and a $F_{1}$ RR field. As a result the 10d metric will be in the form of a warped product and there will be a running dilaton. Moreover, the backreaction of the D7-branes will induce a squashing between the KE base of the Sasaki-Einstein manifold and the fibration [28].

Finite temperature is realized by placing a black hole in the center of the background [8]. The D7-branes extend along the radial direction up to the black hole horizon. Their embedding is described by a constant profile, implementing massless flavor fields in the dual gauge theories. In this work we are interested in switching on a chemical potential for the $\mathrm{U}(1)_{B}$ baryon symmetry, which is the diagonal subgroup of the $\mathrm{U}(1)^{N_{f}}$ flavor symmetry group left over by the smearing of the D7-branes. ${ }^{4}$ The dual picture involves a non-vanishing profile for the temporal component $A_{t}$ of the worldvolume gauge field on the D7-branes $[54,55]$. Through the Chern-Simons coupling, this field can source $F_{3}$ and $\mathrm{H}_{3}$ form fields.

All in all we will be dealing with a general type IIB action given, in Einstein frame, by

$$
\begin{aligned}
S=\frac{1}{2 \kappa_{10}^{2}} & {\left[\int d^{10} x \sqrt{-g}\left(R-\frac{1}{2}(\partial \Phi)^{2}-\frac{e^{-\Phi}}{2} H_{3}^{2}-\frac{e^{2 \Phi}}{2} F_{1}^{2}-\frac{e^{\Phi}}{2} F_{3}^{2}-\frac{1}{4} F_{5}^{2}\right)\right.} \\
& \left.-\int C_{4} \wedge H_{3} \wedge F_{3}\right]+S_{f l},
\end{aligned}
$$

where

$$
S_{f l}=-T_{7} \sum^{N_{f}} \int_{D 7} d^{8} \chi e^{\Phi} \sqrt{-\operatorname{det}\left(\hat{g}+e^{-\Phi / 2} \mathcal{F}\right)}+\mu_{7} \sum^{N_{f}} \int_{D 7} \hat{C}_{q} \wedge\left(e^{-\mathcal{F}}\right)_{8-q}
$$

\footnotetext{
${ }^{4}$ The fundamental fields introduced by means of the D7-branes and the baryonic composites they can form, are thus charged under $\mathrm{U}(1)_{B}$. Moreover, in the generic case we analyze, the vacuum turns out to be charged through a net baryon density, both in the canonical and the grand-canonical ensemble. Fields and composites of the original unflavored models are, of course, uncharged under this $\mathrm{U}(1)_{B}$.
} 
is the contribution of the flavor D7-branes. The gravitational constant and D7-brane tension and charge are, in terms of string parameters

$$
\frac{1}{2 \kappa_{10}^{2}}=\frac{T_{7}}{g_{s}}=\frac{\mu_{7}}{g_{s}}=\frac{1}{(2 \pi)^{7} g_{s}^{2} \alpha^{\prime 4}} .
$$

The smearing procedure $[11,12]$ amounts to a replacement

$$
\sum^{N_{f}} \int_{D 7} X_{8} \rightarrow \int_{M_{10}} X_{8} \wedge \Omega_{2},
$$

for any form $X_{8}$ defined on the brane worldvolume. ${ }^{5}$ Here $\Omega_{2}$ is a form orthogonal to the individual location of the D7-branes. For an arbitrary Sasaki-Einstein space $X_{5}$, it is proportional to the Kähler form $J_{K E}$ of the Kähler-Einstein 4 d basis [28]

$$
g_{s} \Omega_{2}=-2 Q_{f} J_{K E} .
$$

For massless flavors, $Q_{f}$ is a constant encoding the density of D7-branes in the relative quotient space $X_{5} / X_{3}$ with $X_{3}$ the subspace wrapped by each of the branes

$$
Q_{f}=\frac{\operatorname{Vol}\left(X_{3}\right) g_{s} N_{f}}{4 \operatorname{Vol}\left(X_{5}\right)} .
$$

The equations of motion and Bianchi identities that follow from the action (2.1), (2.2) are given in appendix A.

Concerning the metric, we will consider the following ansatz, which includes a family of generalized squashed Sasaki-Einstein manifolds

$$
d s_{10}^{2}=h^{-1 / 2}\left[-b d t^{2}+d x^{i} d x_{i}\right]+h^{1 / 2}\left[b S^{8} F^{2} d \sigma^{2}+S^{2} d s_{K E}^{2}+F^{2}\left(d \tau+A_{K E}\right)^{2}\right],
$$

where the Kähler two-form of the four dimensional base is given in terms of the connection one-form as $J_{K E}=d A_{K E} / 2$. The ansatz (2.7) contains two squashing functions $F(\sigma)$ and $S(\sigma)$ (with dimension of length), whose quotient $F / S$ parameterizes the effect of the flavor backreaction. The dimensionless functions $h(\sigma)$ and $b(\sigma)$ account for the warping and the blackening of the spacetime, respectively. Thus, in particular, an ansatz with $b=1$ is appropriate for the zero-temperature, uncharged solution. We have used the invariance under diffeomorphisms to choose a convenient holographic radial direction, $\sigma$ (with dimension of length ${ }^{-4}$ ), and as we will see, $\sigma \rightarrow-\infty(0)$ in the IR (UV). Given the smearing procedure (2.4) all the functions in our ansatz depend only on the radial variable.

The finite baryon density is dual to a nontrivial worldvolume U(1) gauge field,

$$
\mathcal{F}=2 \pi \alpha^{\prime} A_{t}^{\prime}(\sigma) d \sigma \wedge d t .
$$

A consistent ansatz for the other fields is

$$
\begin{aligned}
& \Phi=\Phi(\sigma), \quad B_{2}=0, \quad F_{1}=Q_{f}\left(d \tau+A_{K E}\right), \quad F_{5}=Q_{c}(1+*) \mathcal{V}\left(X_{5}\right) \\
& F_{3}=F_{123} d x^{1} \wedge d x^{2} \wedge d x^{3}-\frac{J^{\prime} e^{-\Phi}}{S^{4} F^{2}} d t \wedge \Omega_{2}+8 Q_{f} J e^{-\Phi} b F^{2} d t \wedge d \sigma \wedge\left(d \tau+A_{K E}\right) .
\end{aligned}
$$

\footnotetext{
${ }^{5}$ The smearing of the DBI part of the flavor branes is described at length in [28].
} 
In these expressions, $\mathcal{V}\left(X_{5}\right)$ is the volume form of $X_{5}, Q_{c}$ is proportional to the number of colors

$$
Q_{c}=\frac{(2 \pi)^{4} g_{s} \alpha^{2} N_{c}}{\operatorname{Vol}\left(X_{5}\right)}
$$

$F_{123}$ is a constant (of dimension length ${ }^{-1}$ ) which we will show to be related to the baryon density, whereas $J=J(\sigma)$ is a function (of dimension length ${ }^{3}$ ) that describes the effects of the backreaction; ${ }^{6}$ its contribution is dictated by the $C_{6}$ potential

$$
C_{6}=J(\sigma) d x^{1} \wedge d x^{2} \wedge d x^{3} \wedge\left(d \tau+A_{K E}\right) \wedge \Omega_{2},
$$

which is the natural D5 charge sourced on the world-volume of the D7-branes by the gauge field through the last term in (2.2). ${ }^{7}$ In [56] the system without backreacting flavors was studied, and an ansatz for $F_{3}$ was used that only contained the piece proportional to $F_{123}$. However, for our equations of motion to be consistent, we need the presence of the other components; thus, we see that $J(\sigma)$ naturally contains the effects of the backreaction of the flavors.

Inserting the whole ansatz into the 10d equations of motion and Bianchi identities one finally arrives at a system of equations which the reader can find in formulas (A.13)-(A.19). It is possible to describe the whole system in terms of an effective one-dimensional action from which the equations of motion can be derived

$$
S=\frac{\operatorname{Vol}\left(X_{5}\right) V_{1,3}}{2 \kappa_{10}^{2}} \int L_{1 D} d \sigma,
$$

where $V_{1,3}$ denotes the (infinite) integral over the Minkowski coordinates, and

$$
\begin{aligned}
L_{1 D}= & -\frac{1}{2}\left(\log ^{\prime} h\right)^{2}+12\left(\log ^{\prime} S\right)^{2}+8 \log ^{\prime} F \log ^{\prime} S-\frac{1}{2} \Phi^{\prime 2} \\
& +\frac{\log ^{\prime} b}{2}\left(\log ^{\prime} h+8 \log ^{\prime} S+2 \log ^{\prime} F\right)-4 Q_{f}^{2} \frac{J^{\prime 2}}{F^{2} S^{4}} \\
& -\frac{b Q_{c}^{2}}{2 h^{2}}-4 b F^{4} S^{4}+24 b F^{2} S^{6}-\frac{1}{2} F_{123}^{2} e^{\Phi} b h^{2} F^{2} S^{8}-\frac{1}{2} Q_{f}^{2} e^{2 \Phi} b S^{8} \\
& -4 e^{\Phi / 2} F Q_{f} S^{2} \sqrt{-\left(2 \pi \alpha^{\prime} A_{t}^{\prime}\right)^{2}+e^{\Phi} b^{2} F^{2} S^{8}}-32 Q_{f}^{2} e^{-\Phi} b F^{2} J^{2}-8 Q_{f}^{2}\left(2 \pi \alpha^{\prime} A_{t}^{\prime}\right) J .
\end{aligned}
$$

The constraint equation (A.20) is the zero energy condition $H=0$ for the Hamiltonian

$$
H=-L_{1 D}+\sum_{i} \psi_{i}^{\prime} \frac{d L_{1 D}}{d \psi_{i}^{\prime}}, \quad \psi_{i}=\left\{b, h, F, S, \Phi, A_{t}, J\right\}
$$

Since the gauge field $A_{t}$ enters only through its derivative it leads to a "constant of motion". In principle this is a new free parameter which is related to the charge density. However the equations of motion link this to the value of $F_{123}$ in the ansatz for $F_{3}$ in $(2.9)$ as we now show. Let us fix this constant of motion as follows

$$
\frac{\partial L_{1 D}}{\partial A_{t}^{\prime}} \equiv 2 \pi \alpha^{\prime} Q_{c} F_{123}
$$

\footnotetext{
${ }^{6}$ Obviously, $J(\sigma) \neq J_{K E}$.

${ }^{7}$ Notice that, with the smearing, $\sum_{N_{f}} \int_{\mathcal{M}_{8}} \hat{C}_{6} \wedge \mathcal{F} \rightarrow \int_{\mathcal{M}_{10}} C_{6} \wedge \mathcal{F} \wedge \Omega_{2}$.
} 
Solving for $A_{t}^{\prime}$ it gives

$$
2 \pi \alpha^{\prime} A_{t}^{\prime}=\frac{\left(Q_{c} F_{123}+8 Q_{f}^{2} J\right) b F S^{4}}{\sqrt{16 Q_{f}^{2} F^{2} S^{4}+e^{-\Phi}\left(Q_{c} F_{123}+8 Q_{f}^{2} J\right)^{2}}} .
$$

Exactly the same expression is obtained from the equation of motion for the form field $\mathrm{H}_{3}$ (see eq. (A.13)). Thus, by enforcing the integration constant as in (2.16) for consistency, we are putting the system partially on shell. On the other hand, this obscures the analysis when it comes to computing the thermodynamical potentials holographically, since it means that the canonical momentum conjugate to $A_{t}^{\prime}$ was already present in the original Lagrangian. We will comment on this later on.

It is natural to use equation (2.17) to eliminate $A_{t}^{\prime}$ in favor of $F_{123}$ and this is usually done in one of two ways: obtaining the equations of motion from (2.14) and then imposing eq. (2.17), or else, performing a Legendre transformation to the Lagrangian

$$
\tilde{L}_{1 D}=L_{1 D}-\left.\frac{\delta L_{1 D}}{\delta A_{t}^{\prime}} A_{t}^{\prime}\right|_{A_{t}^{\prime}=A_{t}^{\prime}\left(F_{123}\right)},
$$

and then taking the Euler-Lagrange equations from the transformed action. Either way the equations of motion coincide and are given by (A.13)-(A.19) in appendix A.

\section{The perturbative solution}

In the uncharged case $A_{t}^{\prime}=F_{123}=J=0$, the following exact solutions for the functions $b$ and $h$ are readily found [8]: $b=e^{4 r_{0}^{4} \sigma}, h=Q_{c}\left(1-e^{4 r_{0}^{4} \sigma}\right) /\left(4 r_{0}^{4}\right)$, where $r_{0}$ is an integration constant of dimension of length. The black hole horizon is at $\sigma \rightarrow-\infty$ and the extremal limit is reached sending $r_{0} \rightarrow 0$. In terms of a more standard radial coordinate $r$, defined in such a way that $h=R^{4} / r^{4}$ with $R^{4}=Q_{c} / 4$, one gets $b=1-\left(r_{0} / r\right)^{4}$ precisely as for the unflavored $A d S_{5}$ black hole. The horizon radius $r_{h}=r_{0}$ is related to the temperature of the black hole. The whole solution in [8] also depends on the dimensionless combination $\epsilon=Q_{f} e^{\Phi}$, which weighs the backreaction of the D7-branes and, in fact, can be read as a flavor-loop counting parameter in the dual field theory.

Now, $\epsilon$ runs as the dilaton and thus (as a common feature of backreacted D3-D7 setups) it blows up at a finite scale $r_{L P}$ (corresponding to a UV Landau pole in the dual field theory), rendering the supergravity approximation not reliable. Keeping $\epsilon$ small requires restricting the validity range of our solution up to an arbitrary cutoff $r^{*} \ll r_{L P}$, such that

$$
\epsilon_{*}=Q_{f} e^{\Phi_{*}}=\frac{\operatorname{Vol}\left(X_{3}\right)}{16 \pi \operatorname{Vol}\left(X_{5}\right)} \lambda_{*} \frac{N_{f}}{N_{c}} \ll 1,
$$

where $\lambda_{*}=4 \pi g_{s} e^{\Phi_{*}} N_{c} \gg 1, N_{c}, N_{f} \gg 1, \Phi_{*}=\Phi\left(r_{*}\right)$ and we have used (2.11). ${ }^{8}$ In

\footnotetext{
${ }^{8}$ Placing a cutoff in the radial direction does not imply that we are considering a compactified version of our model, which would imply a coupling with $4 \mathrm{~d}$ gravity and a gauging of the flavor symmetry group. The point is that we do not really have a finite volume smooth internal space, but we place "artificially" a cutoff in a geometry that we do not control in the UV (the dilaton diverges). The correct treatment of the boundary conditions and the status of the holographic dictionary in holographic duals of effective field theories is by itself a very interesting line of research that is so far poorly developed.
} 
the uncharged case [8], this condition was used to find an analytic perturbative solution, up to order $\epsilon_{*}^{2}$, for the remaining functions $S, F, \Phi$ appearing in the ansatz. The related integration constants were fixed requiring regularity at the horizon and matching with the $T=0$ solution [28] at the UV cutoff $r_{*}$. The resulting functions thus contained the dimensional (resp. dimensionless) parameters $r_{h}, r_{*}$ (resp. $\epsilon_{*}$ ). The UV cutoff dependent terms resulted to be of the form of both power-like and logarithmic corrections. Formally sending the arbitrary cutoff scale $r_{*}$ to infinity, the first kind of corrections drops out, while the second kind can be handled taking into account that the function $\epsilon$ has a logarithmic running (accounting for the breaking of conformal invariance induced at the quantum level by the massless flavors) such that

$$
\epsilon_{h} \equiv Q_{f} e^{\Phi\left(r_{h}\right)}=\epsilon_{*}\left(1+\epsilon_{*} \log \frac{r_{h}}{r_{*}}\right)+\mathcal{O}\left(\epsilon_{*}^{3}\right) .
$$

This procedure allows to decouple the IR physics from the UV one and to write down a set of solutions containing just $r_{h}$ and $\epsilon_{h}$ as parameters.

In the present charged case, we are going to follow the very same procedure. Here we have a further parameter to deal with: we will call it $\tilde{\delta}$, and we will show that it is related to the dimensionless combination of temperature and baryon chemical potential (or charge density, depending on the thermodynamical ensemble). We will then derive an analytical perturbative solution taking both $\epsilon_{*}$ and $\tilde{\delta}$ to be much smaller than one, deforming the finite temperature flavor backreacted solution obtained in [8].

As a first step, let us introduce a dimensional parameter $\delta$ and consider the following redefinitions

$$
F_{123}=\delta \frac{\sqrt{\epsilon_{*} Q_{f}}}{Q_{c}}, \quad J(\sigma)=\delta \frac{\epsilon_{*}^{3 / 2}}{Q_{f}^{3 / 2}} \tilde{J}(\sigma) .
$$

The reason behind this choice will be clear in a moment. Inserting these expressions into (A.14)-(A.19) and rewriting the dilaton as $\Phi(\sigma)=\Phi_{*}+\phi(\sigma)$, with $\phi\left(\sigma_{*}\right)=0$, one readily arrives at the following system of equations

$$
\begin{aligned}
(\log b)^{\prime \prime} & =4 \epsilon_{*} \delta^{2} \frac{X}{Y}+64 \epsilon_{*}^{2} \delta^{2} e^{-\phi} b F^{2} \tilde{J}^{2}+8 \epsilon_{*}^{2} \delta^{2} e^{-\phi} \frac{\tilde{J}^{\prime 2}}{F^{2} S^{4}}+\epsilon_{*}^{2} \delta^{2} Z \\
(\log h)^{\prime \prime} & =-Q_{c}^{2} \frac{b}{h^{2}}+2 \epsilon_{*} \delta^{2} \frac{X}{Y}+32 \epsilon_{*}^{2} \delta^{2} e^{-\phi} b F^{2} \tilde{J}^{2}+4 \epsilon_{*}^{2} \delta^{2} e^{-\phi} \frac{\tilde{J}^{\prime 2}}{F^{2} S^{4}}+\epsilon_{*}^{2} \delta^{2} \frac{3}{2} Z \\
(\log S)^{\prime \prime} & =-2 b F^{4} S^{4}+6 b F^{2} S^{6}-\epsilon_{*} e^{3 \phi} / 2 \frac{b^{2} F^{3} S^{10}}{Y}-16 \epsilon_{*}^{2} \delta^{2} e^{-\phi} b F^{2} \tilde{J}^{2}-\epsilon_{*}^{2} \delta^{2} \frac{1}{4} Z \\
(\log F)^{\prime \prime}= & 4 b F^{4} S^{4}-\frac{1}{2} \epsilon_{*}^{2} e^{2 \phi} b S^{8}-\epsilon_{*} \delta^{2} \frac{X}{Y}+16 \epsilon_{*}^{2} \delta^{2} e^{-\phi} b F^{2} \tilde{J}^{2}-2 \epsilon_{*}^{2} \delta^{2} e^{-\phi} \frac{\tilde{J}^{\prime 2}}{F^{2} S^{4}}-\epsilon_{*}^{2} \delta^{2} \frac{1}{4} Z \\
(\phi)^{\prime \prime}= & \epsilon_{*}^{2} e^{2 \phi} b S^{8}+2 \epsilon_{*} e^{3 \phi / 2} \frac{b^{2} F^{3} S^{10}}{Y}+2 \epsilon_{*} e^{\phi / 2} F S^{2} Y-32 \epsilon_{*}^{2} \delta^{2} e^{-\phi} b F^{2} \tilde{J}^{2} \\
{\left[\frac{e^{-\phi} \tilde{J}^{\prime}}{S^{4} F^{2}}\right]^{\prime}=} & \frac{-4 \epsilon_{*}^{2} \delta^{2} e^{-\phi} \frac{\tilde{J}^{\prime 2}}{F^{2} S^{4}}+\epsilon_{*}^{2} \delta^{2} \frac{1}{2} Z}{\sqrt{16 F^{2} S^{4}+\delta^{2} e^{-\phi}\left(1+8 \epsilon_{*} \tilde{J}\right)^{2}}}+8 e^{-\phi} b F^{2} \tilde{J}
\end{aligned}
$$


with

$$
\begin{aligned}
X & =\frac{\left(1+8 \epsilon_{*} \tilde{J}\right)^{2} e^{\phi / 2} b^{2} F^{3} S^{10}}{16 F^{2} S^{4}+\delta^{2} e^{-\phi}\left(1+8 \epsilon_{*} \tilde{J}\right)^{2}}, \\
Y & =\sqrt{b^{2} e^{\phi} F^{2} S^{8}-\frac{\delta^{2}\left(1+8 \epsilon_{*} \tilde{J}\right)^{2} b^{2} F^{2} S^{8}}{16 F^{2} S^{4}+\delta^{2} e^{-\phi}\left(1+8 \epsilon_{*} \tilde{J}\right)^{2}}} \\
Z & =\frac{e^{\phi} b h^{2} F^{2} S^{8}}{Q_{c}^{2}} .
\end{aligned}
$$

The constraint equation (A.20) reads

$$
\begin{aligned}
0= & -\frac{1}{2} \log ^{\prime} h \log ^{\prime} b+\frac{1}{2}\left(\log ^{\prime} h\right)^{2}-12\left(\log ^{\prime} S\right)^{2}-4 \log ^{\prime} b \log ^{\prime} S \\
& -\log ^{\prime} b \log ^{\prime} F-8 \log ^{\prime} F \log ^{\prime} S+\frac{1}{2} \phi^{\prime 2} \\
& -\frac{b Q_{c}^{2}}{2 h^{2}}-4 b F^{4} S^{4}+24 b F^{2} S^{6}-\epsilon_{*} \frac{4 e^{3 \phi / 2} b^{2} F^{3} S^{10}}{Y}-\epsilon_{*}^{2} \frac{1}{2} e^{2 \phi} b S^{8} \\
& +\epsilon_{*}^{2} \delta^{2}\left(-32 b e^{-\phi} F^{2} \tilde{J}^{2}+\frac{4 e^{-\phi} \tilde{J}^{\prime 2}}{F^{2} S^{4}}-\frac{1}{2} Z\right) .
\end{aligned}
$$

The system (3.4)-(3.8) allows for a systematic expansion of all the functions in powers series of $\epsilon_{*}$ and $\delta^{2}$. This is essentially the main effect of the scaling relations (3.3). Once all the functions have been solved for, the worldvolume gauge field can be obtained from the following relation

$$
2 \pi \alpha^{\prime} A_{t}^{\prime}=\delta e^{\Phi_{*} / 2} \frac{\left(1+8 \epsilon_{*} \tilde{J}\right) b F S^{4}}{\sqrt{16 F^{2} S^{4}+\delta^{2} e^{-\phi}\left(1+8 \epsilon_{*} \tilde{J}\right)^{2}}}
$$

which is already first order in $\delta$. From this, we also deduce (as previously announced) that $J(\sigma)$ takes the effects of the flavor backreaction into account.

In order to integrate the system (3.4)-(3.8) it is easier to switch to a radial coordinate $y=e^{4 r_{0}^{4} \sigma}$ with $r_{0}$ an arbitrary parameter of dimension of length. The dimensionless parameter $\tilde{\delta}$ referred to as above is then defined as

$$
\tilde{\delta}=\frac{\delta}{4 r_{0}^{3}}
$$

where the factor 4 is introduced in order to make it precisely $\tilde{\delta}=\tilde{d}$ of ref. $[54,55]$ (see also the equation (4.7) in this paper). We keep the greek symbol, however, in order to stress that our parameter is going to be perturbatively small.

Following the same procedure as in the uncharged case, we are now able to provide analytic solutions to the equations above, in a perturbative expansion in $\epsilon_{*}$ and $\tilde{\delta}$. We will skip here the intermediate step where the solutions contain cutoff-dependent terms (this can be found in appendix A up to order $\epsilon_{*} \tilde{\delta}^{2}$ in eqs. (A.22) to (A.26)) and focus on their effective IR expressions. Introducing the IR parameter $\epsilon_{0}=\epsilon\left(r_{0}\right)$ and the radial variable $r$ 
(defined again in such a way that the warp factor keeps the standard AdS form), they read

$$
\begin{aligned}
& h(r)=\frac{R^{4}}{r^{4}}, \\
& b(r)=\left(1-\frac{r_{0}^{4}}{r^{4}}\right)-\frac{\tilde{\delta}^{2} \epsilon_{0}}{2}\left(\left(2-\frac{r_{0}^{4}}{r^{4}}\right)\left(\frac{r_{0}^{2}}{r^{2}}-\log \left[1+\frac{r_{0}^{2}}{r^{2}}\right]\right)\right) \\
& +\frac{\tilde{\delta}^{2} \epsilon_{0}^{2}}{12}\left(17 \frac{r_{0}^{2}}{r^{2}}-9 \frac{r_{0}^{4}}{r^{4}}-\frac{5}{2} \frac{r_{0}^{6}}{r^{6}}-\frac{17}{2}\left(2-\frac{r_{0}^{4}}{r^{4}}\right) \log \left(1+\frac{r_{0}^{2}}{r^{2}}\right)\right)+\ldots, \\
& S(r)=r\left[1+\frac{\epsilon_{0}}{24}+\epsilon_{0}^{2}\left(\frac{9}{1152}-\frac{1}{24} \log \frac{r_{0}}{r}\right)\right. \\
& +\frac{\epsilon_{0} \tilde{\delta}^{2}}{40}\left(3-2 \frac{r^{2}}{r_{0}^{2}}-3\left(1-2 \frac{r^{4}}{r_{0}^{4}}\right) \log \left[1+\frac{r_{0}^{2}}{r^{2}}\right]-\frac{1}{2} G(r)\right) \\
& \left.+\frac{\epsilon_{0}^{2} \tilde{\delta}^{2}}{320}\left(-33+22 \frac{r^{2}}{r_{0}^{2}}+33\left(1-2 \frac{r^{4}}{r_{0}^{4}}\right) \log \left[1+\frac{r_{0}^{2}}{r^{2}}\right]+\frac{11}{2} G(r)\right)+\ldots\right], \\
& F(r)=r\left[1-\frac{\epsilon_{0}}{24}+\epsilon_{0}^{2}\left(\frac{17}{1152}+\frac{1}{24} \log \frac{r_{0}}{r}\right)\right. \\
& +\frac{\epsilon_{0} \tilde{\delta}^{2}}{40}\left(3-22 \frac{r^{2}}{r_{0}^{2}}+5 \frac{r_{0}^{2}}{r^{2}}-3\left(1-2 \frac{r^{4}}{r_{0}^{4}}\right) \log \left[1+\frac{r_{0}^{2}}{r^{2}}\right]+2 G(r)\right) \\
& \left.+\frac{\epsilon_{0}^{2} \tilde{\delta}^{2}}{192}\left(-21+154 \frac{r^{2}}{r_{0}^{2}}-35 \frac{r_{0}^{2}}{r^{2}}+21\left(1-2 \frac{r^{4}}{r_{0}^{4}}\right) \log \left[1+\frac{r_{0}^{2}}{r^{2}}\right]-14 G(r)\right)+\ldots\right] \text {, } \\
& \Phi(r)=\Phi_{0}+\epsilon_{0} \log \frac{r}{r_{0}}-\frac{\epsilon_{0}^{2}}{48}\left(8\left(1+3 \log \frac{r}{r_{0}}\right) \log \frac{r_{0}}{r}-3 \operatorname{Li}_{2}\left[1-\frac{r_{0}^{4}}{r^{4}}\right]\right) \\
& +\frac{\epsilon_{0}^{2} \tilde{\delta}^{2}}{120}\left(26\left(1-\frac{r^{2}}{r_{0}^{2}}\right)-2 \pi-15 \frac{r_{0}^{2}}{r^{2}}+\left(11+18 \frac{r^{4}}{r_{0}^{4}}\right) \log \left[1+\frac{r_{0}^{2}}{r^{2}}\right]-14 \log 2\right. \\
& +G(r)+\ldots), \\
& \tilde{J}(r)=-\frac{r_{0}^{3}}{8}+\ldots, \\
& A_{t}(r)=\frac{r_{0}}{4 \pi \alpha^{\prime}} \tilde{\delta} e^{\Phi_{0} / 2}\left(1-\frac{\epsilon_{0}}{6}\right)\left(1-\frac{r_{0}^{2}}{r^{2}}\right)+\ldots,
\end{aligned}
$$

where $G(r)=2 \pi \frac{r_{0}^{6}}{r^{6}}{ }_{2} F_{1}\left(\frac{3}{2}, \frac{3}{2}, 1,1-\frac{r_{0}^{4}}{r^{4}}\right)$ is an hypergeometric function and $L i_{2}(u) \equiv$ $\sum_{n=1}^{\infty} \frac{u^{n}}{n^{2}}$ is a polylogarithmic function. Notice that $J$ enters at order $\epsilon_{0}^{2}$ in the equations, hence only the leading contribution in $\tilde{J}$ is relevant in the solution.

The above solution must be supplemented with a Jacobian factor for the change of radial coordinate $Y(r)=d \sigma / d r$ which will show up in the coefficient of $d r^{2}$

$$
\begin{aligned}
Y(r)= & \frac{1}{r\left(r^{4}-r_{0}^{4}\right)}+\frac{\tilde{\delta}^{2}}{4 r^{3}\left(r^{4}-r_{0}^{4}\right)^{2}}\left[\epsilon_{0}\left(r_{0}^{2}\left(2 r^{2}-r_{0}^{2}\right)\left(r^{2}+r_{0}^{2}\right)-2 r^{6} \log \left(1+\frac{r_{0}^{2}}{r^{2}}\right)\right)\right. \\
& \left.+\frac{\epsilon_{0}^{2}}{12}\left(-7 r_{0}^{6}+19 r_{0}^{4} r^{2}-34 r_{0}^{2} r^{4}+34 r^{6} \log \left(1+\frac{r_{0}^{2}}{r^{2}}\right)\right)\right]+\ldots
\end{aligned}
$$




\section{Physical properties of the dual plasmas}

\subsection{Thermodynamics}

In the previous section we have derived a solution that, in essence, is a black hole dressed by a set of scalar (dilaton), vector (Maxwell), as well as higher rank tensor fields. Now we are going to extract the thermodynamical properties of the solution, providing, in turn, a first non trivial validity check of the latter by verifying the closure of the standard thermodynamical formulae. As in [8], all quantities are obtained in power series of our perturbative expansion parameters and, therefore, the relevant thermodynamic relations can only be verified up to the relevant order.

To begin with, let us stress that $r_{0}$ does not coincide with the horizon radius $r_{h}$. This radius is defined by $b\left(r_{h}\right)=0+\mathcal{O}\left(\epsilon_{0}^{3}, \tilde{\delta}^{4}\right)$ and is perturbatively shifted by the baryon density $^{9}$

$$
r_{h}=r_{0}\left(1+\frac{\epsilon_{0} \tilde{\delta}^{2}}{8}(1-\log 2)-\frac{\epsilon_{0}^{2} \tilde{\delta}^{2}}{96}(11-17 \log 2)+\ldots\right) .
$$

Notice that $r_{h}>r_{0}$. Notice moreover that

$$
\epsilon_{h} \equiv \epsilon\left(r_{h}\right)=\epsilon_{0}+\mathcal{O}\left(\epsilon_{0}^{3}\right), \quad e^{\Phi_{h}} \equiv e^{\Phi\left(r_{h}\right)}=e^{\Phi_{0}}\left(1+\frac{\epsilon_{0}^{2}}{8} \tilde{\delta}^{2}(1-\log 2)\right)
$$

so that we can trade $\epsilon_{0}$ for $\epsilon_{h}, \tilde{\delta} e^{\Phi_{0}}$ for $\tilde{\delta} e^{\Phi_{h}}$, and so on, in all of our expressions. In particular, looking at (3.18), we get, to leading order in our expansion

$$
A_{t}(r)=\frac{r_{h}}{4 \pi \alpha^{\prime}} \tilde{\delta} e^{\Phi_{h} / 2}\left(1-\frac{\epsilon_{h}}{6}\right)\left(1-\frac{r_{h}^{2}}{r^{2}}\right)
$$

which vanishes at the horizon as required to ensure IR regularity.

The temperature can be computed in the usual way giving

$$
T=\frac{r_{0}}{\pi R^{2}}\left(1-\frac{\epsilon_{h}}{8}\left(1+\tilde{\delta}^{2}\right)-\frac{13}{384} \epsilon_{h}^{2}\left(1-\frac{2}{13} \tilde{\delta}^{2}\right)+\ldots\right)
$$

The entropy density is derived from the horizon's area

$$
s=\frac{1}{2} \frac{\pi^{5}}{\operatorname{Vol}\left(X_{5}\right)} N_{c}^{2} T^{3}\left[1+\frac{1}{2} \epsilon_{h}\left(1+\tilde{\delta}^{2}\right)+\frac{7}{24} \epsilon_{h}^{2}\left(1+\tilde{\delta}^{2}\right)\right] .
$$

As for other thermodynamical variables, this expression, at first order in $\epsilon_{h}$ and $\tilde{\delta}^{2}$, precisely reproduces (for the case of massless flavors) the one found in the probe approximation in $[54,55]$. The $\mathcal{O}\left(\epsilon_{h}^{2}\right)$ terms are instead completely new.

\footnotetext{
${ }^{9}$ In fact, there are two radii $r_{ \pm}$that solve $b\left(r_{ \pm}\right)=0+\mathcal{O}\left(\epsilon_{0}^{3}, \tilde{\delta}^{4}\right)$. This is reminiscent of the case in the Reissner-Nordstrom black hole. The value presented here corresponds to the external radius $r_{h}=r_{+}$, i.e. the event horizon. Numerically we have checked that as $\tilde{\delta}$ increases these two radii approach each other. However, a potentially extremal black hole $r_{-}=r_{+}$cannot be obtained within the range of validity of our solution, which is perturbative in $\tilde{\delta}$.
} 
Concerning the charge density, a proper value is given by the integration constant in (2.16), as its definition coincides precisely with the electric field displacement. In terms of scaling constants we have, to leading order

$$
\frac{d S}{d F_{t \sigma}}=\frac{\operatorname{Vol}\left(X_{5}\right)}{2 \kappa_{10}^{2}} \frac{\partial L_{1 D}}{\partial A_{t}^{\prime}}=\frac{\operatorname{Vol}\left(X_{5}\right)}{(2 \pi)^{7} g_{s}^{2} \alpha^{\prime 4}} 2 \pi \alpha^{\prime} e^{\Phi_{h} / 2} Q_{f} \tilde{\delta} 4 r_{h}^{3},
$$

and making use of (2.6) as well as (4.4) this may be casted in the form

$$
n_{q}=\pi^{7 / 2} \frac{N_{c}^{2}}{\operatorname{Vol}\left(X_{5}\right)^{1 / 2}} \frac{T^{3}}{\sqrt{\lambda_{h}}} \epsilon_{h} \tilde{\delta}\left(1+\frac{3}{8} \epsilon_{h}\right) .
$$

Using (3.1) with $r_{*} \rightarrow r_{h}$ this can be written as

$$
n_{q}=\frac{\pi^{5 / 2}}{16} N_{f} N_{c} \frac{\operatorname{Vol}\left(X_{3}\right)}{\operatorname{Vol}\left(X_{5}\right)^{3 / 2}} \sqrt{\lambda_{h}} T^{3} \tilde{\delta}\left(1+\frac{3}{8} \epsilon_{h}\right) .
$$

At leading order, and for the case $\operatorname{Vol}\left(S^{3}\right)=2 \pi^{2}$ and $\operatorname{Vol}\left(S^{5}\right)=\pi^{3}$, we exactly recover formula (A.11) in $[54,55]{ }^{10}$ In the expressions above, $n_{q}$ is the quark density of the system, related to the baryon density $n_{b}$ by the number of colors $n_{q}=N_{c} n_{b}$.

\subsection{Thermodynamical potentials}

We proceed now to the calculation of the Helmholtz and Gibbs free energies, $\mathcal{F}$ and $\Omega$ respectively. ${ }^{11}$ These can be either directly evaluated starting from the expression for the entropy density and using the standard thermodynamical relations, or they can be deduced holographically. In the latter case they are identified with the (renormalized) on-shell boundary action for the gravity background, evaluated in the corresponding ensemble. Consistency of the solution requires that whatever method is chosen the results are the same. ${ }^{12}$

A quick look at the $1 D$ effective Lagrangian given in (2.14) reveals that on one hand $A_{t}$ is a cyclic coordinate and, on the other hand, $F_{123}$ is a Lagrange multiplier. Amusingly enough, the fact that the equations of motion for $H_{3}$ and $A_{t}$ are consistent with one another imposes (2.16), which is nothing but the statement that, up to constant factors, $F_{123}$ and $A_{t}$ are canonically conjugate variables. Hence, as it stands, $L_{1 D}$ contains both $A_{t}^{\prime}$ and $F_{123}$, hence velocities and momenta. As a consequence the associated action corresponds to neither the canonical nor the grand-canonical ensemble.

\subsubsection{Canonical ensemble}

The Legendre transformed Lagrangian $\tilde{L}_{1 D}$ given in (2.18) is the natural one to describe the system in the canonical ensemble, since it is fully expressed in terms of the baryon density parameter $F_{123}$. Therefore, evaluating the associated action on-shell we should obtain the

\footnotetext{
${ }^{10}$ Beware that $\lambda_{\text {ours }}=4 \pi g_{s} e^{\Phi} N_{c}=2 \lambda_{\text {theirs }}$.

${ }^{11}$ The difference of these quantities with respect to the forms defined in section 2 should be clear from the context.

${ }^{12}$ In the following we will not report the details of the holographic calculations, but we will just give a sketch of the needed ingredients. Needless to say, we have verified that the results deduced from thermodynamical relations agree with those found from holography.
} 
Helmholtz free energy, $\mathcal{F}$. As it is well-known, the action has to be supplemented with the standard Gibbons-Hawking term to deal with a well-posed variational problem. Even with this addition, the evaluation presents divergences which we deal with by subtracting the same quantity evaluated on the Euclidean solution at the same temperature but without a horizon and also with no chemical potential. This procedure is explained in appendix B of [8], where we refer the reader for details. We obtain the Helmholtz free energy density

$$
f=\frac{\mathcal{F}}{V_{3}}=-\frac{1}{8} \frac{\pi^{5}}{\operatorname{Vol}\left(X_{5}\right)} N_{c}^{2} T^{4}\left[1+\frac{1}{2} \epsilon_{h}\left(1-2 \tilde{\delta}^{2}\right)+\frac{1}{6} \epsilon_{h}^{2}\left(1-\frac{7}{2} \tilde{\delta}^{2}\right)\right],
$$

which, consistently, satisfies the thermodynamic relation $-\partial f / \partial T=s$. To check this relation it is very important to consider the dependence of $\epsilon_{h}$ and $\tilde{\delta}$ on $T$.

The logarithmic running of $\epsilon=Q_{f} e^{\Phi(r)}$ (see also eq. (3.2)) and the map between the horizon radius and $T$ give $[8]$

$$
\frac{\partial \epsilon_{h}}{\partial T}=\frac{\epsilon_{h}^{2}}{T}+\mathcal{O}\left(\epsilon_{h}^{3}, \tilde{\delta}^{2}\right) .
$$

Moreover, for $\lambda_{h}(T)=4 \pi g_{s} e^{\Phi_{h}(T)} N_{c}$ one gets by the same token

$$
\frac{\partial \lambda_{h}}{\partial T}=\epsilon_{h} \frac{\lambda_{h}}{T}+\mathcal{O}\left(\epsilon_{h}^{2}, \tilde{\delta}^{2}\right) .
$$

In the canonical ensemble we must keep the physical (dimensional) charge density invariant. From equation (4.8) we see that the dependence of $\tilde{\delta}$ on $T$ at fixed $n_{b}$ comes from solving as

$$
\tilde{\delta}(T)=\frac{n_{b} \alpha}{\sqrt{\lambda_{h}} T^{3}}\left(1+\mathcal{O}\left(\epsilon_{h}\right)+\ldots\right),
$$

with $\alpha$ a $T$-independent constant. Using (4.11) we obtain

$$
\left(\frac{d \tilde{\delta}(T)}{d T}\right)_{n_{b}}=-\frac{\tilde{\delta}}{T}\left(3+\frac{\epsilon_{h}}{2}+\mathcal{O}\left(\epsilon_{h}^{2}\right)\right) .
$$

Using (4.10) and (4.13) it is straightforward to check that $-\partial f / \partial T=s$ with $f$ and $s$ given in (4.9) and (4.5) respectively. This is a strong proof of consistency.

Next we can evaluate the ADM energy density of the plasma, again subtracting the contribution from the zero temperature and baryon density setup to get rid of the divergences. Following appendix B in [8] the final result is

$$
\varepsilon=\frac{E_{A D M}}{V_{3}}=\frac{3}{8} \frac{\pi^{5}}{\operatorname{Vol}\left(X_{5}\right)} N_{c}^{2} T^{4}\left[1+\frac{1}{2} \epsilon_{h}\left(1+2 \tilde{\delta}^{2}\right)+\frac{1}{3} \epsilon_{h}^{2}\left(1+\frac{7}{4} \tilde{\delta}^{2}\right)\right]
$$

which satisfies the relation $\varepsilon=f+s T$. From here, and taking again into account (4.10) and (4.13), we obtain the heat capacity at fixed baryon number density

$$
c_{V, n_{q}}=\left(\frac{\partial \varepsilon}{\partial T}\right)_{V, n_{b}}=\frac{3}{2} \frac{\pi^{5}}{\operatorname{Vol}\left(X_{5}\right)} N_{c}^{2} T^{3}\left[1+\frac{1}{2} \epsilon_{h}\left(1-\tilde{\delta}^{2}\right)+\frac{1}{24} \epsilon_{h}^{2}\left(11-7 \tilde{\delta}^{2}\right)\right] .
$$




\subsubsection{Grand-canonical ensemble}

We would like to obtain the thermodynamic quantities corresponding to the grandcanonical ensemble. It would be tempting to think that the correct Lagrangian density to use here is the original one $L_{1 D}$ in (2.14). However, this is not the case. As mentioned before, the fact that we have identified the canonical momentum conjugate to $A_{t}^{\prime}$ with $F_{123}$ interferes with the Legendre transform, since we see that this parameter already appears in $L_{1 D}$. Were it not for this fact, the following inverse Legendre transform

$$
\tilde{\tilde{L}}_{1 D}=\tilde{L}_{1 D}-\left.\frac{\partial \tilde{L}_{1 D}}{\partial F_{123}} F_{123}\right|_{F_{123}\left(A_{t}\right)},
$$

where $F_{123}\left(A_{t}^{\prime}\right)$ comes from solving

$$
A_{t}^{\prime}=\frac{\partial \tilde{L}_{1 D}}{\partial F_{123}}
$$

would bring us back to the original lagrangian $\tilde{\tilde{L}}_{1 D}=L_{1 D}$. However, notice the presence of the term with $F_{123}^{2}$ in (2.14), which upon (4.16) will change sign. Therefore, the relevant Lagrangian for the computation of the Gibbs free energy is given in (2.14) with a sign flip in the term with $F_{123}^{2}$. Doing that, and following the same steps as for $f$, we arrive at the result

$$
\omega=\frac{\Omega}{V_{3}}=-p=-\frac{1}{8} \frac{\pi^{5}}{\operatorname{Vol}\left(X_{5}\right)} N_{c}^{2} T^{4}\left[1+\frac{1}{2} \epsilon_{h}\left(1+2 \tilde{\delta}^{2}\right)+\frac{1}{6} \epsilon_{h}^{2}\left(1+\frac{7}{2} \tilde{\delta}^{2}\right)\right],
$$

where $p$ is the pressure. Now we can compare this expression with the one for the energy density given in (4.14). The interaction energy, given by

$$
\frac{\varepsilon-3 p}{T^{4}}=\frac{\pi^{2} N_{c}^{2}}{16 \operatorname{Vol}\left(X_{5}\right)} \epsilon_{h}^{2},
$$

is just the same as in the uncharged case. Hence, charge density of massless flavors does not contribute to the breaking of conformal invariance. This is not unexpected in field theory and, in a dual gravity picture, it is the same as what happens in the case of the Reissner-Nordström AdS black hole, where the presence of a net charge does not spoil the relation $\varepsilon=3 p$.

We now would like to check the thermodynamic relations in the present ensemble. Notice that the interpretation of $\tilde{\delta}$ has changed and, henceforth, also its behavior with the temperature. The reason is that the physical parameter to fix in the grand-canonical ensemble is the dimensional chemical potential, $\mu$. The difference among the two thermodynamical potentials gives

$$
\mu n_{q}=f-\omega=\frac{\pi^{5}}{4 \operatorname{Vol}\left(X_{5}\right)} N_{c}^{2} T^{4} \epsilon_{h} \tilde{\delta}^{2}\left(1+\frac{7}{12} \epsilon_{h}\right) .
$$

Using (4.7) we can extract the chemical potential ${ }^{13}$

$$
\mu=\frac{\pi^{3 / 2}}{4 \operatorname{Vol}\left(X_{5}\right)^{1 / 2}} \sqrt{\lambda_{h}} T \tilde{\delta}\left(1+\frac{5}{24} \epsilon_{h}\right)
$$

\footnotetext{
${ }^{13}$ Using the particular value of $\operatorname{Vol}\left(X_{5}\right)=\pi^{3}$ we get the same expression at leading order in $\epsilon_{h}$ as in eq. (A.10) in [54, 55] after identifying $\tilde{\mu}=\tilde{\delta} / 2$.
} 
Now, the value of the world-volume gauge field in the UV (i.e. at $r \gg r_{h}$ ) is (see eq. (4.3))

$$
A_{t, U V}=\frac{1}{4} \sqrt{\frac{\lambda_{h}}{\pi \operatorname{Vol}\left(X_{5}\right)}} \pi^{2} T \tilde{\delta}\left(1-\frac{\epsilon_{h}}{24}\right) .
$$

Comparing the variation of the grand potential with respect to $A_{t, U V}$

$$
\frac{\delta \omega}{\delta A_{t, U V}}=-\pi^{7 / 2} \frac{N_{c}^{2}}{\operatorname{Vol}\left(X_{5}\right)^{1 / 2}} \frac{T^{3}}{\sqrt{\lambda_{h}}} \epsilon_{h} \tilde{\delta}\left(1+\frac{5}{8} \epsilon_{h}\right),
$$

with the relation

$$
\frac{\delta \omega}{\delta \mu}=-n_{b},
$$

and taking into account formula (4.7), we get the connection between the UV value of the gauge field and the chemical potential

$$
A_{t, U V}=\mu\left(1-\frac{1}{4} \epsilon_{h}\right) .
$$

Note that this differs at subleading order from the result $A_{t, U V}=\mu$ as obtained in $[54,55]$; this is thus an effect of the backreaction of the flavors.

From (4.21) we see that for fixed $\mu, \tilde{\delta}$ acquires now a dependence on $T$ like

$$
\tilde{\delta}(T)=\frac{4}{\pi^{2}} \sqrt{\frac{\pi \operatorname{Vol}\left(X_{5}\right)}{\lambda_{h}(T)}} \frac{\mu}{T}\left(1-\frac{5}{24} \epsilon_{h}\right) .
$$

Using (4.10) again and (4.26) we now get

$$
\left(\frac{d \tilde{\delta}}{d T}\right)_{\mu}=-\frac{\tilde{\delta}}{T}\left(1+\frac{\epsilon_{h}}{2}+\mathcal{O}\left(\epsilon_{h}^{2}\right)\right) .
$$

With this scaling (and equation (4.10)) it is easy to check that the thermodynamic relation $-\partial \omega / \partial T=s$ holds at the required order. Using again (4.27) we can obtain readily the heat capacity at fixed chemical potential

$$
c_{V, \mu}=\left(\frac{\partial \varepsilon}{\partial T}\right)_{V, \mu}=\frac{3}{2} \frac{\pi^{5}}{\operatorname{Vol}\left(X_{5}\right)} N_{c}^{2} T^{3}\left[1+\frac{1}{2} \epsilon_{h}\left(1+\tilde{\delta}^{2}\right)+\frac{1}{24} \epsilon_{h}^{2}\left(11+7 \tilde{\delta}^{2}\right)\right],
$$

from which we can extract the speed of sound

$$
c_{s}^{2}=\frac{s}{c_{V, \mu}}=\frac{1}{3}\left(1-\frac{1}{6} \epsilon_{h}^{2}\right) .
$$

The parameter $\delta_{c}=1-3 c_{s}^{2}$ is related to the breaking of conformality as before. As it happens for the interaction energy, it does not receive corrections from the presence of finite baryon density. Using the arguments in [8], this suggests that the bulk viscosity is not affected by the presence of a finite baryon density on the system. ${ }^{14}$

\footnotetext{
${ }^{14}$ There is some mismatch between our results for the speed of sound and the heat capacities and corresponding results found in the literature in the probe approximation. The precise closure of the thermodynamical relations and the consistent independence of the conformality breaking effects from the baryon chemical potential in the massless flavored case, let us be confident of the correctness of our results.
} 
Susceptibilities. The "quark" susceptibility 15 is

$$
\chi=-\frac{\partial^{2} \omega}{\partial \mu^{2}}=\frac{\partial n_{q}}{\partial \mu}=\frac{\pi \operatorname{Vol}\left(X_{3}\right)}{4 \operatorname{Vol}\left(X_{5}\right)} N_{f} N_{c} T^{2}\left(1+\frac{1}{6} \epsilon_{h}\right) .
$$

At leading order this matches with the result obtained in the probe approximation in [57] for the flavored $\mathcal{N}=4 \mathrm{SYM}$ case (where $\operatorname{Vol}\left(X_{3}\right)=2 \pi^{2}, \operatorname{Vol}\left(X_{5}\right)=\pi^{3}$ ). The other three susceptibilities are

$$
-\frac{\partial^{2} \omega}{\partial T^{2}}=\frac{\partial s}{\partial T}=\frac{3 \pi^{5}}{2 \operatorname{Vol}\left(X_{5}\right)} N_{c}^{2} T^{2}\left(1+\frac{1}{2} \epsilon_{h}+\frac{11}{24} \epsilon_{h}^{2}\right)+\frac{\pi \operatorname{Vol}\left(X_{3}\right)}{4 \operatorname{Vol}\left(X_{5}\right)} N_{f} N_{c} \mu^{2}\left(1+\frac{1}{6} \epsilon_{h}\right),
$$

and

$$
-\frac{\partial^{2} \omega}{\partial \mu \partial T}=-\frac{\partial^{2} \omega}{\partial T \partial \mu}=\frac{\pi \operatorname{Vol}\left(X_{3}\right)}{2 \operatorname{Vol}\left(X_{5}\right)} N_{f} N_{c} \mu T\left(1+\frac{1}{6} \epsilon_{h}\right) .
$$

The determinant of the susceptibility matrix equals $\chi C$ where

$$
C=\frac{3 \pi^{5}}{2 \operatorname{Vol}\left(X_{5}\right)} N_{c}^{2} T^{2}\left(1+\frac{1}{2} \epsilon_{h}+\frac{11}{24} \epsilon_{h}^{2}\right)-\frac{3 \pi \operatorname{Vol}\left(X_{3}\right)}{4 \operatorname{Vol}\left(X_{5}\right)} N_{f} N_{c} \mu^{2}\left(1+\frac{1}{6} \epsilon_{h}\right) .
$$

The second term is parameterically smaller than the first one, thus the theory is thermodynamically stable.

\subsection{Probe parton energy loss}

In order to estimate how the finite charge density or chemical potential influences the energy loss of an energetic probe parton traveling through the plasma, we will make use of the results in [58-60]. In this approach, the parton looses energy through bremsstrahlung due to its interactions with the strongly coupled medium. The amount of energy loss, which is ultimately the cause of the jet quenching in the strongly coupled plasma, is encoded in a transport coefficient termed $\hat{q}$, the "jet-quenching parameter". The latter can be derived, using the eikonal approximation at high energy, as the coefficient of $L^{2}$ in an almost light-like Wilson loop with dimensions $L^{-} \gg L$. The Wilson loop is easily calculated in string theory.

In [61] a formula to extract the jet quenching parameter from a general dual gravity background was derived. ${ }^{16}$ On our solution, rewritten in the variable $y=\exp \left(4 r_{0}^{4} \sigma\right)$, the formula gives

$$
\hat{q}^{-1}=\pi \alpha^{\prime} \int_{0}^{1} e^{-\frac{\Phi}{2}} \frac{\sqrt{g_{y y}}}{g_{x x} \sqrt{g_{x x}+g_{t t}}} d y=\pi \int_{0}^{1} \frac{1}{4 r_{0}^{4} y e^{\frac{\Phi}{2}}} \sqrt{\frac{b}{1-b}} h F S^{4} d y,
$$

where in particular $g_{x x}$ is the metric coefficient in the spatial direction involved in the trajectory of the parton. For our purposes, it will be enough to analyze the result up to order $\epsilon_{h} \tilde{\delta}^{2}$, which reads

$$
\hat{q}=\frac{\pi^{3} \sqrt{\lambda_{h}} \Gamma\left(\frac{3}{4}\right)}{\sqrt{\operatorname{Vol}\left(X_{5}\right)} \Gamma\left(\frac{5}{4}\right)} T^{3}\left[1+\frac{2+\pi}{8} \epsilon_{h}\left(1+c \tilde{\delta}^{2}\right)\right],
$$

\footnotetext{
${ }^{15}$ As usual, "quark" is an abuse of language for "fundamental matter".

${ }^{16}$ We took into account a different factor of $\sqrt{2}$ between the definitions in $[59,60]$ and $[61]$.
} 
with a positive constant $c=0.867565 .^{17}$

\subsubsection{Comparison schemes}

Formula (4.35), without any prescription to compare different theories, would imply that a finite charge density (or chemical potential) increases the jet quenching. This conclusion would depend on considering the jet quenching parameter in theories with different numbers of degrees of freedom. From a phenomenological perspective, it is more useful to compare theories keeping the number of degrees of freedom fixed. In order to fully appreciate the effects of flavors at finite charge density, we will compare our flavored theory with the unflavored $\mathcal{N}=4 \mathrm{SYM}$.

For an estimate of the number of degrees of freedom, the most used observables are the entropy density (4.5), and the energy density (4.14). In large $N_{c}$ theories, it makes sense to keep one of these two quantities fixed by either fixing the number of colors $N_{c}$ and changing the temperature $T$, or the other way around. First, let us consider the fixed $N_{c}$, varying $T$ comparison scheme, used for example in [62]. For the sake of definitiveness, we also keep the energy density fixed; the qualitative result which will follow is unchanged if we fix the entropy density instead. We get

$$
T_{f}=T_{u}\left[1-\frac{\epsilon_{h}}{8}\left(1+2 \tilde{\delta}^{2}\right)\right]
$$

where the subindex refers to either the flavored or the $u$ nflavored $\mathcal{N}=4$ theory. Plugging this result in formula (4.35) we read

$$
\frac{\hat{q}_{f}}{\hat{q}_{u}} \sim \sqrt{\frac{\lambda_{f}}{\lambda_{u}}}\left[1+\frac{\pi-1}{8} \epsilon_{h}\left(1-0.719 \tilde{\delta}^{2}\right)\right]
$$

where both 't Hooft couplings $\lambda_{f}, \lambda_{u}$ are evaluated at the horizon. Since $N_{c}$ is fixed, ${ }^{18}$ if we keep the coupling $\alpha_{s}$ fixed, we obtain the result that while the jet quenching parameter is enhanced by the addition of flavors [8], the finite charge density (or chemical potential) actually reduces the enhancement. ${ }^{19}$

This qualitative result is unchanged if, following strictly [62], we also allow for the variation of $\alpha_{s}$. The latter can be adjusted in such a way that the force between two external quarks at the screening length $L_{c, u}$ of the unflavored plasma, $\alpha_{q q}=3 L_{c, u}^{2} V^{\prime}\left(L_{c, u}\right) / 4$, is kept fixed. The potential $V(L)$ is calculated numerically by standard formulas, reported for example in [63]. We calculate $\alpha_{q q}$ for different values of $N_{f}$ (equivalently, $\epsilon_{h}$ ) and $\tilde{\delta}$. At zero charge density, as we increase $N_{f}, \alpha_{q q}$ increases, so in order to keep it fixed the coupling $\lambda_{f}$ must be decreased. ${ }^{20}$ But the effect is small, so as anticipated we get in (4.37)

\footnotetext{
${ }^{17}$ The constant $c$ is related to the result of the integral

$$
\int_{0}^{1} \frac{-(8-3 \sqrt{1-y}+2 y)(1-y)+\sqrt{1-y}(3+2 y)(2 \operatorname{arctanh}[\sqrt{1-\mathrm{y}}]+\log \mathrm{y})}{(1-y)^{9 / 4} \sqrt{y}} d y .
$$

${ }^{18}$ Remember that $\lambda \sim \alpha_{s} N_{c}$.

${ }^{19}$ Remember that the term in $\epsilon_{h} \tilde{\delta}^{2}$ is by definition a perturbation of the one in $\epsilon_{h}$.

${ }^{20}$ This corrects a statement in footnote 20 of [8].
} 
that the jet quenching parameter is enhanced by the addition of flavors. Along the same lines, if we switch on the charge density, we find that this enhancement is reduced.

We can now analyze what happens if we use the alternative comparison scheme introduced in [8], where the temperature $T$ is kept fixed and the number of degrees of freedom is kept constant by varying the number of colors $N_{c}$. By fixing the energy density (4.14) we get

$$
N_{c, f}=N_{c, u}\left[1-\frac{1}{4} \epsilon_{h}\left(1+2 \tilde{\delta}^{2}\right)\right] .
$$

Keeping fixed also the coupling constant $\alpha_{s}$ for simplicity, we obtain

$$
\frac{\hat{q}_{f}}{\hat{q}_{u}} \sim 1+\frac{\pi+1}{8} \epsilon_{h}\left(1+0.594 \tilde{\delta}^{2}\right) .
$$

Thus, while again the overall effect of the flavors is to enhance the jet quenching, in this case the charge density actually increases the enhancement. The result is qualitatively the same if we keep fixed the entropy density (4.5) instead of the energy density.

To summarize, while in all the cases the effect of fundamental flavors is to enhance the jet quenching [8], the net effect of a finite charge density (or chemical potential) actually depends on how we compare different theories. Namely, we found that the charge density reduces (resp. increases) the enhancement of the jet quenching due to flavors in the varying $T$, fixed $N_{c}$ (resp. fixed $T$, varying $N_{c}$ ) comparison scheme. We currently have no intuitive explanation for this behavior.

\subsection{Remarks on "optical" properties}

The solution presented in this paper is dual, in the hydrodynamic limit, to a charged relativistic fluid. Recently, it has been observed in [64,65] that, generically, this kind of fluids has interesting uncommon optical properties, such as negative refractive index, and exhibits exotic phenomena, like the propagation of additional light waves in certain frequency regimes. It is understood that one can speak of "propagation of light" by thinking of gauging the global U(1) charge: a gravity solution such as the one presented above (which accounts instead for a global U(1)) allows anyway for the calculation of the current correlators (which can be treated as external, non-dynamical ones, in linear response theory) involved in the optical analysis. Alternatively, one could maybe think about "optical properties" of baryon charge waves (e.g. dispersion relations).

To be concrete, in [64] it was shown that every relativistic fluid with finite charge density $\rho$ ( $n_{q}$ in our solution), having a dispersive pole in the (transverse) current retarded correlator $^{21}$

$$
G_{T}(\omega, k) \sim \frac{i \omega \mathcal{B}}{i \omega-\mathcal{D} k^{2}}+P(0, k),
$$

displays negative refraction, i.e. the phase and group velocities have opposite directions, for small enough frequencies $\omega^{2}<4 \pi q^{2} \mathcal{B}$, where $q$ is the $\mathrm{U}(1)$ gauge coupling, if the term $P(0, k)$ can be discarded. The latter requirement was verified by explicit gravity

\footnotetext{
${ }^{21}$ Clearly, here $\omega, k$ are the frequency and wave vector.
} 


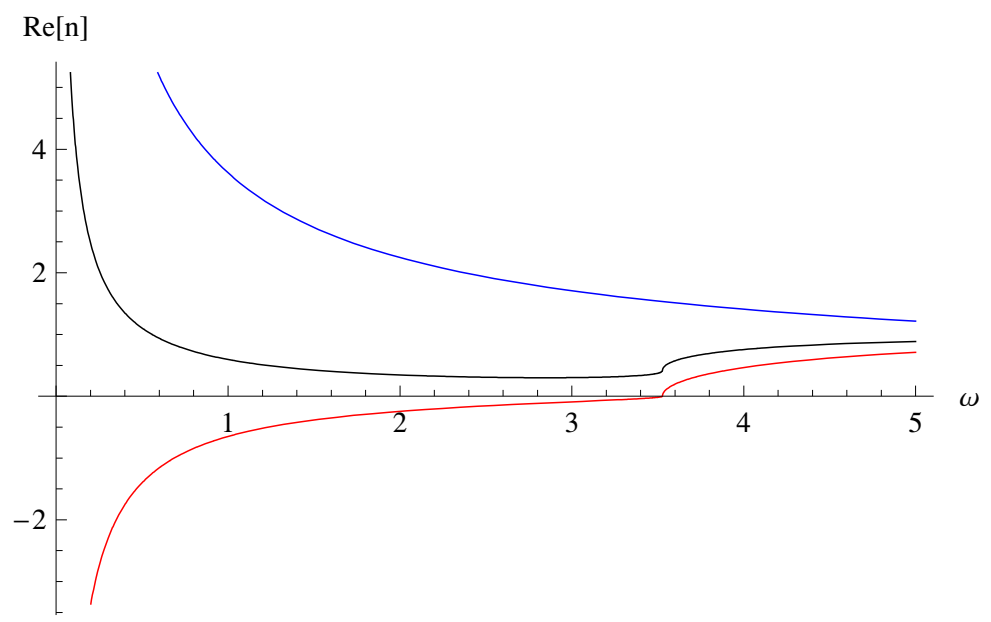

Figure 1. The real part of the two refractive indexes $n_{1}$ (upper line) and $n_{2}$ (bottom line), with the effective one $n_{\text {eff }}$ (central line), as functions of the frequency (we are using unit temperature). The plot refers to the $\mathcal{N}=4$ theory with the following parameter choice: $N_{c}=100, N_{f}=20, \lambda_{h}=$ $50, q=1, \tilde{\delta}=0.1$.

calculations in [64-66]. In formula (4.40) one has

$$
\mathcal{B}=\frac{\rho^{2}}{\varepsilon+p}, \quad \mathcal{D}=\frac{\eta}{\varepsilon+p}
$$

where $\eta$ is the shear viscosity of the fluid. In the case at hand ${ }^{22}$

$$
\mathcal{B}=\frac{\pi \operatorname{Vol}\left(X_{3}\right)}{8 \operatorname{Vol}\left(X_{5}\right)} N_{f} N_{c} T^{2} \epsilon_{h} \tilde{\delta}^{2}, \quad \mathcal{D}=\frac{1}{4 \pi T}-\frac{\epsilon_{h} \tilde{\delta}^{2}}{8 \pi T}\left(1+\frac{1}{12} \epsilon_{h}\right)
$$

Thus, assuming again that the term $P(0, k)$ does not affect the result, the charged D3-D7 Plasmas would exhibit negative refraction for

$$
\omega_{c}^{2}<q^{2} \frac{\pi^{2} \operatorname{Vol}\left(X_{3}\right)}{2 \operatorname{Vol}\left(X_{5}\right)} N_{f} N_{c} T^{2} \epsilon_{h} \tilde{\delta}^{2} .
$$

Actually, in a medium with large spatial dispersion, there is the possibility of the propagation of two "light waves" for each incident wave, due to the fact that the dispersion relation admits two different solutions ${ }^{23}[65]$

$$
n_{1,2}^{2}=\frac{\mathcal{D}+\frac{i}{\omega} \pm \sqrt{\frac{16 i \pi q^{2} \mathcal{B D}+\omega(\mathcal{D} \omega-i)^{2}}{\omega^{3}}}}{2 \mathcal{D}}
$$

This effect is due to the coupling of the incident wave with "exciton" quasi-particles in the medium, which in the dual gravity description are quasi-normal modes. One of the two indexes should correspond to negative refraction for small $\omega$, while the other one is

\footnotetext{
${ }^{22}$ We make use of the relation $\eta / s=1 / 4 \pi$ [67].

${ }^{23}$ Again, assuming that $P(0, k)$ is ineffective.
} 
connected to the existence of an additional light wave. The propagation of both waves in certain frequency regimes could be inferred from the study of an "effective refractive index"

$$
n_{\mathrm{eff}}=\frac{1+n_{1} n_{2}}{n_{1}+n_{2}} .
$$

The results for the real part of the two refractive indexes and the effective one are summarized in figure $1 .^{24}$ One of the two indexes displays negative refraction for $\omega<\omega_{c}$, with $\omega_{c}$ given precisely by (4.43). ${ }^{25}$ The interpolation of the effective index $n_{\text {eff }}$ from one refractive index at small frequencies to the other one at large frequencies, which can be seen in the plot, signals that in the intermediate regime both light waves actually propagate.

In conclusion, the outcome has very similar features to the ones found in the analysis of the RN black hole cases in [64-66]. Thus, we seem to see the expected features already at the level of hydrodynamics. It would be very interesting to study the Green functions directly in the gravity setting to understand whether this behavior is confirmed and to what extent the hydrodynamic approximation is reliable.

\section{Conclusions}

Following the strategy initiated in [8], in the present paper we give the crank another turn, by introducing a new deformation that encodes holographically the backreaction of charged degrees of freedom at finite density. Following the same logic we provide an analytic solution which is perturbative in the deformation parameters, and explicitly write it down up to second order in both $\epsilon_{h}$ and $\tilde{\delta}$. Moreover it is an effective solution. This means that only IR quantities can be reliably computed from it. In this case, the IR energy scale, $\mu_{I R}$, is set by the temperature, and all results have to be understood as being correct up to terms of order $\mu_{I R} / \mu_{U V}=T / \mu_{U V}$ where $\mu_{U V}$ is an ultraviolet cutoff energy scale above which the ultraviolet completion sets in. Still a lot of phenomenologically interesting prediction can be made both for thermodynamic quantities as well as for transport coefficients. We have shown that the thermodynamics is consistent, and we are in a stable phase. So far, nothing can be said about phase transitions. The solution contains two horizons, and has the potential to exhibit an extremal solution that would allow to explore the zero temperature - finite chemical potential axis. However such a systematic search of the phase space in the $(T, \mu)$ plane lies outside the domain of validity of the analytic solution presented here. It would require the use of numerical techniques, along the lines of the recent work [68] and certainly constitutes a natural continuation of the present work.

Concerning transport phenomena, we have studied the jet quenching parameter and found that the effect of the net baryon density is positive or negative depending on the way one compares different theories with and without flavor. In the present context this effect

\footnotetext{
${ }^{24}$ We thank A. Amariti, D. Forcella and A. Mariotti for their crucial observations on the results.

${ }^{25}$ The imaginary part of one of the two indexes, $\operatorname{Im}\left[n_{2}\right]$, would be negative for $\omega<\omega_{c}$. This means that we have to choose, for $\omega<\omega_{c}$, the negative branch of the square root of $n_{2}^{2}$ in (4.44), which results in the negative refraction. We also find a particular value of $\omega_{p}>\omega_{c}$ for which the two indexes exchange (i.e. the continuous functions are made by gluing the two indexes at $\omega_{p}$ ). This is probably an artifact of Mathematica.
} 
is subdominant as compared to the one driven by the presence of flavor. Again, the fate of this at higher finite values of the charge density can only be established numerically, and its importance is evident from the fact that this is one of the few windows to phenomenology for a rather wide class of models. It is also worth comparing this result with the information one can obtain from the evaluation of the drag force.

Last but not least, we have analyzed the possibility of exotic optical phenomena in the case that the global U(1) could be gauged and treated as the electromagnetic one. Following [64, 65], we have checked the presence of a negative refractive index and the propagation of additional light waves in certain frequency regimes. Our analysis concerns the expectations for the optical properties, based on hydrodynamic considerations. It would be very interesting to go beyond the hydrodynamic approximation by the explicit gravity calculation of the relevant retarded Green functions.

\section{Acknowledgments}

We would like to thank Paolo Benincasa, Angel Paredes and Alfonso V. Ramallo for useful discussions. We are indebted with Antonio Amariti, Davide Forcella and Alberto Mariotti for their explanations concerning the optical properties of relativistic charged fluids. J.T. is thankful to the Front of Galician-speaking Scientists for encouragement.

The research of F.B. and A.L.C. is supported by European Community's Seventh Framework Programme (FP7/2007-2013) under grant agreements n. 253937 and n. 253534, respectively. J.T. is supported by the Netherlands Organization for Scientific Research (NWO) under the FOM Foundation research program.

J.M. and J.T. are supported by the MICINN and FEDER (grant FPA2008-01838), the Spanish Consolider-Ingenio 2010 Programme CPAN (CSD2007-00042), and the Xunta de Galicia (Conselleria de Educacion and grant INCITE09-206-121-PR). Part of their research was done while visiting the Erwin Schroedinger Institut Vienna and the Kavli Institute Beijing. They want to thank both the ESI-Vienna and the KITPC Beijing for hospitality and finantial support (this last under grant KJCX2.YW.W10 of the Chinese Academy of Sciences).

F.B. and A.L.C. would like to thank the Italian students, parents, teachers and scientists for their activity in support of public education and research.

\section{A Technical details}

\section{A.1 Equations of motion from the ten dimensional action}

The equations of motion derived from the action $S$ as given in (2.1) and (2.2) are [53] $]^{26}$

$$
\begin{aligned}
R_{M N}-\frac{1}{2} g_{M N} R= & \frac{1}{2}\left(\partial_{M} \Phi \partial_{N} \Phi-\frac{1}{2} g_{M N} \partial_{P} \Phi \partial^{P} \Phi\right)+\frac{1}{2} e^{2 \Phi}\left(F_{1 M} F_{1 N}-\frac{1}{2} g_{M N} F_{1}^{2}\right) \\
& +\frac{1}{4} e^{\Phi}\left(F_{3 M P Q} F_{3 N}{ }^{P Q}-g_{M N} F_{3}^{2}\right)+\frac{1}{4} e^{-\Phi}\left(H_{3 M P Q} H_{3 N}{ }^{P Q}-g_{M N} H_{3}^{2}\right)
\end{aligned}
$$

\footnotetext{
${ }^{26}$ In our conventions $F_{p}^{2}=\frac{1}{p !}\left(F_{p}\right)^{a_{1} a_{2} \ldots a_{p}}\left(F_{p}\right)_{a_{1} a_{2} \ldots a_{p}}$ (also for $H_{3}$ ). Notice moreover that self-duality of $F_{5}$ has been imposed.
} 


$$
\begin{gathered}
+\frac{1}{96} F_{5 M P Q R S} F_{5 N}{ }^{P Q R S}+\frac{2 \kappa^{2}}{\sqrt{-g}} \frac{\delta S_{f l}}{\delta g^{M N}}, \\
\square \Phi=e^{2 \Phi} F_{1}^{2}+\frac{1}{2} e^{\Phi} F_{3}^{2}-\frac{1}{2} e^{-\Phi} H_{3}^{2}-\frac{2 \kappa^{2}}{\sqrt{-g}} \frac{\delta S_{f l}}{\delta \Phi} \\
d\left(e^{2 \Phi} * F_{1}\right)=-e^{\Phi} H_{3} \wedge * F_{3}-\frac{1}{24} \mathcal{F}^{4} \wedge \Omega_{2}, \\
d\left(e^{\Phi} * F_{3}\right)=-H_{3} \wedge F_{5}+\frac{1}{6} \mathcal{F}^{3} \wedge \Omega_{2}, \\
d\left(* F_{5}\right)=d F_{5}=H_{3} \wedge F_{3}-\frac{1}{2} \mathcal{F}^{2} \wedge \Omega_{2}, \\
d\left(e^{-\Phi} * H_{3}\right)=e^{\Phi} F_{1} \wedge * F_{3}-F_{5} \wedge F_{3}+e^{\Phi} \frac{\delta}{\delta \mathcal{F}} \sqrt{-\operatorname{det}\left(\hat{g}+e^{-\Phi / 2} \mathcal{F}\right)} \delta^{(2)}(D 7),
\end{gathered}
$$

where the last term in the equation for $H_{3}$ has to be meant as an eight-form: in particular $\delta^{(2)}(D 7)$ is a short-hand notation for the form which arises taking the derivative w.r.t. $\mathcal{F}$ of the smeared DBI action, where $\sqrt{-\operatorname{det}\left(\hat{g}+e^{-\Phi / 2} \mathcal{F}\right)} d^{8} \chi$ is replaced by

$$
\sqrt{-\operatorname{det}\left(g+e^{-\Phi / 2} \mathcal{F}\right)}\left|\Omega_{2}\right| d^{10} x .
$$

The expression for the modulus of the $\Omega_{2}$ form will be given in a moment.

The Bianchi identities read

$$
\begin{aligned}
d F_{1} & =-g_{s} \Omega_{2}, \\
d F_{3} & =H_{3} \wedge F_{1}-\mathcal{F} \wedge \Omega_{2}, \\
d H_{3} & =0
\end{aligned}
$$

Finally, the Bianchi identity and EOM for the brane field $\mathcal{F}$ are

$$
\begin{gathered}
d \mathcal{F}=H_{3}, \\
d\left(e^{\Phi} \frac{\delta}{\delta \mathcal{F}} \sqrt{-\operatorname{det}\left(\hat{g}+e^{-\Phi / 2} \mathcal{F}\right)} \delta^{(2)}(D 7)\right)=d(\cdots),
\end{gathered}
$$

where the dots represent the terms from the WZ part of the flavor action.

After inserting the ansatz introduced in (2.9) the equations of motion (A.3) (A.4) and (A.5) as well as the Bianchi identities (A.8) and (A.10) are automatically satisfied. The 1.h.s. of the equation of motion (A.6) for $H_{3}$ vanishes identically upon imposing the ansatz, and from its right hand side (explicitly rewritten taking into account eq. (A.7), with $\left|\Omega_{2}\right|=4 Q_{f} h^{-1 / 2} S^{-2}$ ) we can solve for $A_{t}^{\prime}$ to obtain

$$
2 \pi \alpha^{\prime} A_{t}^{\prime}=\frac{\left(Q_{c} F_{123}+8 Q_{f}^{2} J\right) b F S^{4}}{\sqrt{16 Q_{f}^{2} F^{2} S^{4}+e^{-\Phi}\left(Q_{c} F_{123}+8 Q_{f}^{2} J\right)^{2}}} .
$$

This is an important relation, and interestingly enough, it automatically solves the equation of motion for $A_{t}$. This is because (A.12) is precisely the radial derivative of (A.6). The Bianchi identity (A.11) is identically null. 
The Bianchi identity for $F_{3}$ (A.9) gives a nontrivial relation between $A_{t}^{\prime}$ and $J$. Using (A.13) this can be expressed as an independent equation for $J$ which we have added to the list below as eq. (A.19).

Finally we come to the Einstein-dilaton equations of motion (A.1) and (A.2)

$$
\begin{aligned}
(\log b)^{\prime \prime}= & 4 Q_{f} \frac{X}{Y}+64 Q_{f}^{2} e^{-\Phi} b F^{2} J^{2}+8 Q_{f}^{2} e^{-\Phi} \frac{J^{\prime 2}}{F^{2} S^{4}}+Z \\
(\log h)^{\prime \prime}= & -Q_{c}^{2} \frac{b}{h^{2}}+2 Q_{f} \frac{X}{Y}+32 Q_{f}^{2} e^{-\Phi} b F^{2} J^{2}+4 Q_{f}^{2} e^{-\Phi} \frac{J^{\prime 2}}{F^{2} S^{4}}+\frac{3}{2} Z \\
(\log S)^{\prime \prime}= & -2 b F^{4} S^{4}+6 b F^{2} S^{6}-Q_{f} e^{3 \Phi / 2} \frac{b^{2} F^{3} S^{10}}{Y}-16 Q_{f}^{2} e^{-\Phi} b F^{2} J^{2}-\frac{1}{4} Z \\
(\log F)^{\prime \prime}= & 4 b F^{4} S^{4}-\frac{1}{2} Q_{f}^{2} e^{2 \Phi} b S^{8}-Q_{f} \frac{X}{Y}+16 Q_{f}^{2} e^{-\Phi} b F^{2} J^{2}-2 Q_{f}^{2} e^{-\Phi} \frac{J^{\prime 2}}{F^{2} S^{4}} \\
& -\frac{1}{4} Z \\
(\Phi)^{\prime \prime}= & Q_{f}^{2} e^{2 \Phi} b S^{8}+2 Q_{f} e^{3 \Phi / 2} \frac{b^{2} F^{3} S^{10}}{Y}+2 Q_{f} e^{\Phi / 2} F S^{2} Y-32 Q_{f}^{2} e^{-\Phi} b F^{2} J^{2}+\frac{1}{2} Z \\
& -4 Q_{f}^{2} e^{-\Phi} \frac{J^{\prime 2}}{F^{2} S^{4}}, \\
{\left[\frac{e^{-\Phi} J^{\prime}}{S^{4} F^{2}}\right]^{\prime}=} & \frac{\left(Q_{c} F_{123}+8 Q_{f}^{2} J\right) b F S^{4}}{\sqrt{16 Q_{f}^{2} F^{2} S^{4}+e^{-\Phi}\left(Q_{c} F_{123}+8 Q_{f}^{2} J\right)^{2}}}+8 e^{-\Phi} b F^{2} J
\end{aligned}
$$

and the constraint that comes from fixing the reparameterization gauge invariance in the radial variable reads

$$
\begin{aligned}
0= & -\frac{1}{2} \log ^{\prime} h \log ^{\prime} b+\frac{1}{2}\left(\log ^{\prime} h\right)^{2}-12\left(\log ^{\prime} S\right)^{2}-4 \log ^{\prime} b \log ^{\prime} S \\
& -\log ^{\prime} b \log ^{\prime} F-8 \log ^{\prime} F \log ^{\prime} S+\frac{1}{2} \Phi^{\prime 2} \\
& -\frac{b Q_{c}^{2}}{2 h^{2}}-4 b F^{4} S^{4}+24 b F^{2} S^{6}-\frac{1}{2} Z-\frac{1}{2} b e^{2 \Phi} Q_{f}^{2} S^{8} \\
& -\frac{4 b^{2} e^{3 \Phi / 2} F^{3} Q_{f} S^{10}}{Y}-32 b e^{-\Phi} F^{2} Q_{f}^{2} J^{2}+\frac{4 e^{-\Phi} Q_{f}^{2} J^{\prime 2}}{F^{2} S^{4}} .
\end{aligned}
$$

In these expressions we defined

$$
X=\left(2 \pi \alpha^{\prime} A_{t}^{\prime}\right)^{2} e^{\Phi / 2} F S^{2} ; \quad Y=\sqrt{-\left(2 \pi \alpha^{\prime} A_{t}^{\prime}\right)^{2}+b^{2} e^{\Phi} F^{2} S^{8}} ; \quad Z=F_{123}^{2} e^{\Phi} b h^{2} F^{2} S^{8} .
$$

\section{A.2 Solution with explicit UV cutoff}

The solution with explicit dependence on the position of the radial cutoff $r_{*}$ to first order in $\epsilon_{*}$ reads

$$
\begin{aligned}
b(r) & =1-\frac{r_{0}^{4}}{r^{4}}-\frac{\tilde{\delta}^{2} \epsilon_{*}}{2}\left(2-\frac{r_{0}^{4}}{r^{4}}\right)\left(\left(1-\frac{r^{2}}{r_{*}^{2}}\right) \frac{r_{0}^{2}}{r^{2}}-\log \left[\frac{1+\frac{r_{0}^{2}}{r^{2}}}{1+\frac{r_{0}^{2}}{r_{*}^{2}}}\right]\right)+\mathcal{O}\left(\epsilon_{*}^{2}\right), \\
\frac{S(r)}{r} & =1+\frac{\epsilon_{*}}{36} \frac{3 r_{*}^{4}-r^{4}-r_{0}^{4}}{2 r_{*}^{4}-r_{0}^{4}}
\end{aligned}
$$




$$
\begin{aligned}
& +\frac{\tilde{\delta}^{2} \epsilon_{*}}{40}\left(\frac{r_{*}^{4}\left(6-4 \frac{r^{2}}{r_{0}^{2}}\right)+\frac{r_{*}^{2}}{r_{0}^{2}}\left(12 r^{4}-6 r_{0}^{4}\right)+\left(2 r^{2} r_{0}^{2}-6 r^{4}\right)+\frac{r_{0}^{2}}{r_{*}^{2}}\left(r_{0}^{4}-2 r^{4}\right)}{2 r_{*}^{4}-r_{0}^{4}}\right. \\
& \left.-\frac{4 r_{*}^{4}-r_{0}^{4}}{r_{*}^{4}} \frac{r_{*}^{2}}{r_{0}^{2}} \frac{G_{0}(r)}{G_{0}\left(r_{*}\right)}-3\left(1-2 \frac{r^{4}}{r_{0}^{4}}\right) \log \left[\frac{1+\frac{r_{0}^{2}}{r^{2}}}{1+\frac{r_{0}^{2}}{r_{*}^{2}}}\right]\right)+\mathcal{O}\left(\epsilon_{*}^{2}\right), \\
& \frac{F(r)}{r}=1-\frac{\epsilon_{*}}{36} \frac{3 r_{*}^{4}+r^{4}-2 r_{0}^{4}}{2 r_{*}^{4}-r_{0}^{4}} \\
& +\frac{\tilde{\delta}^{2} \epsilon_{*}}{40}\left(\frac{r_{*}^{4}\left(6-44 \frac{r^{2}}{r_{0}^{2}}+10 \frac{r_{0}^{2}}{r^{2}}\right)+r_{*}^{2} r^{2}\left(12 \frac{r^{2}}{r_{0}^{2}}-6 \frac{r_{0}^{2}}{r^{2}}\right)-r^{4}\left(6-22 \frac{r_{0}^{2}}{r^{2}}+5 \frac{r_{0}^{6}}{r^{6}}\right)}{2 r_{*}^{4}-r_{0}^{4}}\right. \\
& \left.-\frac{r_{0}^{2}}{r_{*}^{2}} \frac{2 r^{4}-r_{0}^{4}}{2 r_{*}^{4}-r_{0}^{4}}+\frac{16 r_{*}^{4}-4 r_{0}^{4}}{r_{*}^{4}} \frac{r_{*}^{2}}{r_{0}^{2}} \frac{G_{0}(r)}{G_{0}\left(r_{*}\right)}-3\left(1-2 \frac{r^{4}}{r_{0}^{4}}\right) \log \left[\frac{1+\frac{r_{0}^{2}}{r^{2}}}{1+\frac{r_{0}^{2}}{r_{*}^{2}}}\right]\right) \\
& +\mathcal{O}\left(\epsilon_{*}^{2}\right), \\
& \Phi(r)=\Phi_{*}+\epsilon_{*} \log \left(\frac{r}{r_{*}}\right)+\mathcal{O}\left(\epsilon_{*}^{2}\right), \\
& J(r)=-\frac{r_{0}^{3}}{8} \frac{r_{*}^{4}-r^{4}}{r_{*}^{4}+r_{0}^{4}}+\mathcal{O}\left(\epsilon_{*}\right),
\end{aligned}
$$

Open Access. This article is distributed under the terms of the Creative Commons Attribution Noncommercial License which permits any noncommercial use, distribution, and reproduction in any medium, provided the original author(s) and source are credited.

\section{References}

[1] BRAHMS collaboration, I. Arsene et al., Quark Gluon Plasma an Color Glass Condensate at RHIC? The perspective from the BRAHMS experiment, Nucl. Phys. A 757 (2005) 1 [nucl-ex/0410020] [SPIRES].

[2] B.B. Back et al., The PHOBOS perspective on discoveries at RHIC, Nucl. Phys. A 757 (2005) 28 [nucl-ex/0410022] [SPIRES].

[3] STAR collaboration, J. Adams et al., Experimental and theoretical challenges in the search for the quark gluon plasma: The STAR collaboration's critical assessment of the evidence from RHIC collisions, Nucl. Phys. A 757 (2005) 102 [nucl-ex/0501009] [SPIRES].

[4] PHENIX collaboration, K. Adcox et al., Formation of dense partonic matter in relativistic nucleus nucleus collisions at RHIC: Experimental evaluation by the PHENIX collaboration, Nucl. Phys. A 757 (2005) 184 [nucl-ex/0410003] [SPIRES].

[5] The ALICE collaboration, K. Aamodt et al., Elliptic flow of charged particles in $\mathrm{Pb}-\mathrm{Pb}$ collisions at 2.76 TeV, arXiv:1011.3914 [SPIRES].

[6] ATLAS collaboration, G. Aad et al., Observation of a Centrality-Dependent Dijet Asymmetry in Lead-Lead Collisions at sqrt $(S(N N))=2.76 \mathrm{TeV}$ with the ATLAS Detector at the LHC, Phys. Rev. Lett. 105 (2010) 252303 [arXiv:1011.6182] [SPIRES]. 
[7] ALICE collaboration, K. Aamodt et al., Suppression of Charged Particle Production at Large Transverse Momentum in Central Pb-Pb Collisions at $\sqrt{s_{N N}}=2.76 \mathrm{TeV}$, Phys. Lett. B 696 (2011) 30 [arXiv:1012.1004] [SPIRES].

[8] F. Bigazzi et al., D3-D7 quark-gluon Plasmas, JHEP 11 (2009) 117 [arXiv:0909.2865] [SPIRES].

[9] F. Bigazzi, A.L. Cotrone and J. Tarrio, Hydrodynamics of fundamental matter, JHEP 02 (2010) 083 [arXiv:0912.3256] [SPIRES].

[10] F. Bigazzi and A.L. Cotrone, An elementary stringy estimate of transport coefficients of large temperature QCD, JHEP 08 (2010) 128 [arXiv:1006.4634] [SPIRES].

[11] F. Bigazzi, R. Casero, A.L. Cotrone, E. Kiritsis and A. Paredes, Non-critical holography and four-dimensional CFT's with fundamentals, JHEP 10 (2005) 012 [hep-th/0505140] [SPIRES].

[12] R. Casero, C. Núñez and A. Paredes, Towards the string dual of $N=1$ SQCD-like theories, Phys. Rev. D 73 (2006) 086005 [hep-th/0602027] [SPIRES].

[13] C. Núñez, A. Paredes and A.V. Ramallo, Unquenched flavor in the gauge/gravity correspondence, Adv. High Energy Phys. 2010 (2010) 196714 [arXiv:1002.1088] [SPIRES].

[14] R. Casero, A. Paredes and J. Sonnenschein, Fundamental matter, meson spectroscopy and non-critical string/gauge duality, JHEP 01 (2006) 127 [hep-th/0510110] [SPIRES].

[15] E. Nakano, S. Teraguchi and W.-Y. Wen, Drag Force, Jet Quenching and AdS/QCD, Phys. Rev. D 75 (2007) 085016 [hep-ph/0608274] [SPIRES].

[16] K. Kajantie, T. Tahkokallio and J.-T. Yee, Thermodynamics of AdS/QCD, JHEP 01 (2007) 019 [hep-ph/0609254] [SPIRES].

[17] G. Bertoldi, F. Bigazzi, A.L. Cotrone and J.D. Edelstein, Holography and Unquenched quark-gluon Plasmas, Phys. Rev. D 76 (2007) 065007 [hep-th/0702225] [SPIRES].

[18] A.L. Cotrone, J.M. Pons and P. Talavera, Notes on a SQCD-like plasma dual and holographic renormalization, JHEP 11 (2007) 034 [arXiv:0706.2766] [SPIRES].

[19] Y.-h. Gao, W.-s. Xu and D.-f. Zeng, Viscosity and jet quenching from holographic model, arXiv:0707.0817 [SPIRES].

[20] O. Lorente-Espin and P. Talavera, A silence black hole: Hawking radiation at the Hagedorn temperature, JHEP 04 (2008) 080 [arXiv:0710.3833] [SPIRES].

[21] M. Mia, K. Dasgupta, C. Gale and S. Jeon, Five Easy Pieces: The Dynamics of Quarks in Strongly Coupled Plasmas, Nucl. Phys. B 839 (2010) 187 [arXiv:0902.1540] [SPIRES].

[22] M. Mia, K. Dasgupta, C. Gale and S. Jeon, The Double Life of Thermal QCD, arXiv:0902.2216 [SPIRES].

[23] G. Bertoldi and C. Hoyos-Badajoz, Stability of linear dilaton black holes at the Hagedorn temperature, JHEP 08 (2009) 078 [arXiv:0903.3431] [SPIRES].

[24] M. Mia and C. Gale, Jet quenching and the gravity dual, Nucl. Phys. A 830 (2009) 303c-306c [arXiv:0907.4699] [SPIRES].

[25] E. Caceres, R. Flauger and T. Wrase, Hagedorn Systems from Backreacted Finite Temperature $N_{f}=2 N_{c}$ Backgrounds, arXiv:0908.4483 [SPIRES]. 
[26] M. Mia, K. Dasgupta, C. Gale and S. Jeon, Toward Large-N Thermal QCD from Dual Gravity: The Heavy Quarkonium Potential, Phys. Rev. D 82 (2010) 026004 [arXiv: 1004.0387] [SPIRES].

[27] M. Mia, K. Dasgupta, C. Gale and S. Jeon, Heavy Quarkonium Melting in Large-N Thermal QCD, Phys. Lett. B 694 (2011) 460 [arXiv:1006.0055] [SPIRES].

[28] F. Benini, F. Canoura, S. Cremonesi, C. Núñez and A.V. Ramallo, Unquenched flavors in the Klebanov-Witten model, JHEP 02 (2007) 090 [hep-th/0612118] [SPIRES].

[29] A. Paredes, On unquenched $N=2$ holographic flavor, JHEP 12 (2006) 032 [hep-th/0610270] [SPIRES].

[30] F. Benini, F. Canoura, S. Cremonesi, C. Núñez and A.V. Ramallo, Backreacting Flavors in the Klebanov-Strassler Background, JHEP 09 (2007) 109 [arXiv:0706.1238] [SPIRES].

[31] R. Casero, C. Núñez and A. Paredes, Elaborations on the String Dual to $N=1 S Q C D$, Phys. Rev. D 77 (2008) 046003 [arXiv:0709.3421] [SPIRES].

[32] E. Caceres, R. Flauger, M. Ihl and T. Wrase, New Supergravity Backgrounds Dual to $N=1$ SQCD-like Theories with $N_{f}=2 N_{c}$, JHEP 03 (2008) 020 [arXiv:0711.4878] [SPIRES].

[33] F. Canoura, P. Merlatti and A.V. Ramallo, The supergravity dual of 3d supersymmetric gauge theories with unquenched flavors, JHEP 05 (2008) 011 [arXiv:0803.1475] [SPIRES].

[34] F. Bigazzi, A.L. Cotrone, C. Núñez and A. Paredes, Heavy quark potential with dynamical flavors: a first order transition, Phys. Rev. D 78 (2008) 114012 [arXiv:0806.1741] [SPIRES].

[35] F. Bigazzi, A.L. Cotrone and A. Paredes, Klebanov-Witten theory with massive dynamical flavors, JHEP 09 (2008) 048 [arXiv: 0807. 0298] [SPIRES].

[36] C. Hoyos-Badajoz, C. Núñez and I. Papadimitriou, Comments on the String dual to $N=1$ SQCD, Phys. Rev. D 78 (2008) 086005 [arXiv:0807.3039] [SPIRES].

[37] D. Arean, P. Merlatti, C. Núñez and A.V. Ramallo, String duals of two-dimensional (4,4) supersymmetric gauge theories, JHEP 12 (2008) 054 [arXiv:0810.1053] [SPIRES].

[38] F. Bigazzi, A.L. Cotrone and A. Paredes, Phase transitions in large- $N_{c}$ heavy quark potentials, Nucl. Phys. Proc. Suppl. 192-193 (2009) 134 [arXiv:0810.4018] [SPIRES].

[39] F. Bigazzi, A.L. Cotrone, A. Paredes and A. Ramallo, Non chiral dynamical flavors and screening on the conifold, Fortsch. Phys. 57 (2009) 514 [arXiv:0810.5220] [SPIRES].

[40] J. Gaillard and J. Schmude, On the geometry of string duals with backreacting flavors, JHEP 01 (2009) 079 [arXiv:0811.3646] [SPIRES].

[41] A.V. Ramallo, J.P. Shock and D. Zoakos, Holographic flavor in $N=4$ gauge theories in $3 d$ from wrapped branes, JHEP 02 (2009) 001 [arXiv:0812.1975] [SPIRES].

[42] F. Bigazzi, A.L. Cotrone, A. Paredes and A.V. Ramallo, The Klebanov-Strassler model with massive dynamical flavors, JHEP 03 (2009) 153 [arXiv: 0812.3399] [SPIRES].

[43] F. Bigazzi, A.L. Cotrone, A. Paredes and A.V. Ramallo, Screening effects on meson masses from holography, JHEP 05 (2009) 034 [arXiv:0903.4747] [SPIRES].

[44] O.C. Gurdogan, Walking solutions in the string background dual to $N=1 S Q C D$-like theories, Annals Phys. 325 (2010) 535 [arXiv:0906.2429] [SPIRES]. 
[45] J. Gaillard and J. Schmude, The lift of type IIA supergravity with D6 sources: M-theory with torsion, JHEP 02 (2010) 032 [arXiv:0908.0305] [SPIRES].

[46] C. Núñez, M. Piai and A. Rago, Wilson Loops in string duals of Walking and Flavored Systems, Phys. Rev. D 81 (2010) 086001 [arXiv:0909.0748] [SPIRES].

[47] D. Arean, E. Conde and A.V. Ramallo, Gravity duals of 2d supersymmetric gauge theories, JHEP 12 (2009) 006 [arXiv:0909.3106] [SPIRES].

[48] D. Elander, Glueball Spectra of SQCD-like Theories, JHEP 03 (2010) 114 [arXiv:0912.1600] [SPIRES].

[49] D. Arean, E. Conde, A.V. Ramallo and D. Zoakos, Holographic duals of SQCD models in low dimensions, JHEP 06 (2010) 095 [arXiv: 1004.4212] [SPIRES].

[50] J. Gaillard, D. Martelli, C. Núñez and I. Papadimitriou, The warped, resolved, deformed conifold gets flavoured, Nucl. Phys. B 843 (2011) 1 [arXiv: 1004.4638] [SPIRES].

[51] J. Schmude, Comments on the distinction between color- and flavor-branes and new D3-D7 solutions with eight supercharges, arXiv:1007.1201 [SPIRES].

[52] E. Conde and J. Gaillard, Kutasov-like duality from D5-branes wrapping hyperbolic cycles, arXiv: 1011.1451 [SPIRES].

[53] F. Benini, A chiral cascade via backreacting D7-branes with flux, JHEP 10 (2008) 051 [arXiv:0710.0374] [SPIRES].

[54] S. Kobayashi, D. Mateos, S. Matsuura, R.C. Myers and R.M. Thomson, Holographic phase transitions at finite baryon density, JHEP 02 (2007) 016 [hep-th/0611099] [SPIRES].

[55] D. Mateos, S. Matsuura, R.C. Myers and R.M. Thomson, Holographic phase transitions at finite chemical potential, JHEP 11 (2007) 085 [arXiv:0709.1225] [SPIRES].

[56] H.-Y. Chen, K. Hashimoto and S. Matsuura, Towards a Holographic Model of Color-Flavor Locking Phase, JHEP 02 (2010) 104 [arXiv: 0909.1296] [SPIRES].

[57] R.C. Myers, A.O. Starinets and R.M. Thomson, Holographic spectral functions and diffusion constants for fundamental matter, JHEP 11 (2007) 091 [arXiv: 0706.0162] [SPIRES].

[58] R. Baier, Y.L. Dokshitzer, A.H. Mueller, S. Peigne and D. Schiff, Radiative energy loss and $p_{T}$-broadening of high energy partons in nuclei, Nucl. Phys. B 484 (1997) 265 [hep-ph/9608322] [SPIRES].

[59] H. Liu, K. Rajagopal and U.A. Wiedemann, Calculating the jet quenching parameter from AdS/CFT, Phys. Rev. Lett. 97 (2006) 182301 [hep-ph/0605178] [SPIRES].

[60] F. D'Eramo, H. Liu and K. Rajagopal, Transverse Momentum Broadening and the Jet Quenching Parameter, Redux, arXiv:1006.1367 [SPIRES].

[61] N. Armesto, J.D. Edelstein and J. Mas, Jet quenching at finite 't Hooft coupling and chemical potential from AdS/CFT, JHEP 09 (2006) 039 [hep-ph/0606245] [SPIRES].

[62] S.S. Gubser, Comparing the drag force on heavy quarks in $N=4$ super-Yang-Mills theory and QCD, Phys. Rev. D 76 (2007) 126003 [hep-th/0611272] [SPIRES].

[63] Y. Kinar, E. Schreiber and J. Sonnenschein, $Q$ anti- $Q$ potential from strings in curved spacetime: Classical results, Nucl. Phys. B 566 (2000) 103 [hep-th/9811192] [SPIRES].

[64] A. Amariti, D. Forcella, A. Mariotti and G. Policastro, Holographic Optics and Negative Refractive Index, arXiv:1006.5714 [SPIRES]. 
[65] A. Amariti, D. Forcella and A. Mariotti, Additional Light Waves in Hydrodynamics and Holography, arXiv: 1010.1297 [SPIRES].

[66] X.-H. Ge, K. Jo and S.-J. Sin, Hydrodynamics of RN AdS $S_{4}$ black hole and Holographic Optics, JHEP 03 (2011) 104 [arXiv: 1012.2515] [SPIRES].

[67] P. Kovtun, D.T. Son and A.O. Starinets, Viscosity in strongly interacting quantum field theories from black hole physics, Phys. Rev. Lett. 94 (2005) 111601 [hep-th/0405231] [SPIRES].

[68] O. DeWolfe, S.S. Gubser and C. Rosen, A holographic critical point, arXiv:1012.1864 [SPIRES]. 